\title{
Quel impact attendre de la généralisation de la complémentaire santé d'entreprise sur le taux de non couverture en France ? Une simulation à partir de l'enquête Santé et Protection Sociale 2012
}

\author{
Aurélie Pierre (Irdes) \\ Florence Jusot (PSL, Université Paris Dauphine, Leda-Legos \& Irdes)
}

\begin{abstract}
:
En France, malgré le rôle prépondérant de l'assurance maladie obligatoire au financement des dépenses de santé (75,9\% en moyenne en 2012), l'accès aux soins est fortement dépendant de la possession d'un contrat d'assurance complémentaire santé, et de sa qualité, dont 5\% de la population reste dépourvu en 2012. La généralisation de l'accès à une complémentaire santé se trouve ainsi au cœur de la stratégie nationale de santé énoncée en 2013 par Marisol Touraine et la première mesure négociée dans le cadre de l'Accord national interprofessionnelle (ANI) de janvier 2013 consistera à généraliser la complémentaire santé d'entreprise et améliorer sa portabilité au $1^{\text {er }}$ janvier 2016. Cet article propose d'étudier les effets attendus de ce dispositif sur la généralisation de la complémentaire santé en France et ses conséquences en termes de bien-être d'une part, ainsi que sur les inégalités d'accès à la complémentaire santé d'autre part. A partir de l'Enquête Santé et Protection Sociale 2012, il s'agit i/ de simuler l'impact de l'introduction de l'ANI sur la proportion d'individus qui resteront non couverts par une complémentaire en population générale et selon l'état de santé, le statut socioéconomique, les préférences à l'égard du temps et du risque et le motif de non couverture; ii/ d'étudier l'évolution des caractéristiques des personnes non couvertes toutes choses égales par ailleurs avant et après l'introduction de 1'ANI. Nous montrons que le taux de non couverts, estimé à $4 \%$ après la généralisation de la complémentaire santé d'entreprise et à 3,7\% en tenant compte de la portabilité, restera toujours plus élevé pour les populations les plus fragiles, et en particulier les chômeurs. L'ANI va en revanche diminuer le lien toutes choses égales par ailleurs entre la non couverture et les préférences à' l'égard du temps et du risque. Cette mesure risque donc de contrarier la non couverture par choix sans pour éliminer la non couverture pour raisons financières.
\end{abstract}

Mots clés : Complémentaire santé, ANI, inégalités d'accès aux soins

Les auteurs remercient Nicolas Célant pour son aide précieuse sur l'exploitation des données de la complémentaire santé dans l'Enquête Santé et Protection Sociale, ainsi que Mireille Elbaum pour ses échanges concernant le dispositif de l'ANI et l'accès à la complémentaire santé des jeunes âgés de 16 à 30 ans. 


\section{Introduction}

En France, malgré le rôle prépondérant de l'assurance maladie obligatoire au financement des dépenses de santé (76 \% en moyenne en 2013 ; Zaidman et Roussel, 2014), l'accès aux soins est fortement dépendant de la possession d'un contrat de couverture complémentaire santé et de sa qualité (Buchmueller et al., 2004 ; Dourgnon et al., 2012 ; Jusot, 2013). Face à ce constat, plusieurs dispositifs ont été mis en place par le gouvernement pour favoriser l'accès à une complémentaire santé pour les plus précaires (CMU-C en $2000^{1}$, Aide à l'acquisition d'une complémentaire santé en $2005^{2}$ ) mais également pour soutenir le développement des complémentaires santé d'entreprise (exonérations de charges sociales et fiscales introduites en 1985), de sorte que le taux de personnes qui ne bénéficient pas d'une telle couverture n'a cessé de diminuer depuis les années 1990 (Perronnin et al., 2011). Néanmoins, l'accès à une complémentaire santé demeure très inégalitaire et en $2012,5 \%$ de la population déclarant toujours ne pas être couvert par une complémentaire santé. En effet, $6 \%$ des Français bénéficient gratuitement d'une couverture en raison de la faiblesse de leurs revenus par le biais de la Couverture maladie universelle complémentaire (CMU-C), 53\% bénéficient d'un contrat à titre individuel et 35\% bénéficient d'un contrat collectif, c'est-à-dire souscrite par le biais de leur employeur ou de l'employeur d'un membre de leur ménage (Célant et al., 2014 ; Jusot, 2014). C'est pourquoi la généralisation de l'accès à une complémentaire santé se trouve au cœur de la stratégie nationale de santé énoncée par Marisol Touraine le 23 septembre 2013, au coté de l'objectif de réduction des inégalités sociales de santé (Touraine, 2014). La première mesure, négociée dans le cadre de l'Accord national interprofessionnelle (ANI) de janvier 2013, consiste à généraliser à l'ensemble des salariés du secteur privé ou d'un particulier la complémentaire santé collective partiellement financée par les employeurs, et sa portabilité au $1^{\text {er }}$ janvier 2016.

Au-delà des questions concernant les conséquences de ce nouveau dispositif sur la répartition du risque assurantiel entre le marché individuel et celui du collectif (Franc et Pierre, 2013), on peut s'interroger sur sa capacité à généraliser la couverture par une complémentaire santé en France et à réduire les inégalités face à la complémentaire santé. En effet, celui-ci ne concernera que les salariés du secteur privé ou d'un particulier, qui constitue une population aujourd'hui largement couverte dans la mesure où ces derniers bénéficient déjà pour $64 \%$ d'entre eux d'un contrat de complémentaire santé collective (Célant et al. 2014), qui offre par ailleurs de meilleures garanties pour un moindre coût que les contrats de complémentaire santé individuelle (Perronnin et al., 2012 ; Garnero et Le Palud, 2013). Plusieurs travaux français ont en outre montré que le revenu était l'élément le plus déterminant de la

\footnotetext{
${ }^{1}$ La Couverture maladie universelle complémentaire (CMU-C) a été instaurée par la loi du 27 juillet 1999. Elle donne accès de droit à une couverture complémentaire gratuite aux ménages les plus pauvres. Au 1er janvier 2014, elle concerne les ménages dont le niveau de vie était inférieur à $716 €$ par mois pour une personne seule en métropole.

${ }^{2}$ Le dispositif de l'Aide à l'acquisition d'une complémentaire santé (ACS), mis en place au ler janvier 2005 est une aide financière qui s'applique uniquement aux contrats individuels «responsables». Elle s'adresse aux ménages ayant des ressources jusqu'à $35 \%$ supérieures au plafond de l'octroi de la CMU-C en 2014 (entre $716 €$ et $967 €$ par mois pour une personne seule en métropole) et son montant varie en fonction de l'âge des personnes couvertes par le contrat.
} 
décision de souscrire un contrat de complémentaire santé individuel (Buchmueller et Couffinhal, 2004 ; Saliba et Ventelou, 2007 ; Arnould et Vidal, 2008 ; Grignon et Kambia-Chopin, 2009 ; Jusot et al., 2012), l'état de santé ayant un effet relativement modéré. De fait, le premier motif évoqué par les individus non couverts concerne le coût des contrats (Célant et al., 2014) qui, relativement au revenu, peut s'avérer très important pour les ménages les plus modestes (Kambia-Chopin et al., 2008 ; Jusot et al., 2012). Ainsi, alors même que l'objectif annoncé du gouvernement est de permettre à tous les Français de bénéficier d'une complémentaire santé de qualité, la généralisation de la complémentaire santé d'entreprise, qui exclue de facto les individus qui ne sont pas présents sur le marché du travail, et donc pour une large part, les personnes modestes ou les plus malades, risque donc au contraire de renforcer les inégalités d'accès, de coût et de niveau de couverture au détriment de ceux qui auraient le plus besoin d'une complémentaire santé.

Par ailleurs, on peut s'interroger sur les conséquences en termes de bien-être de l'introduction d'une obligation d'assurance pour l'ensemble des salariés du secteur privé ou d'un particulier. En effet, la non couverture étant majoritairement expliquée par l'inaccessibilité financière de la complémentaire santé pour les ménages les plus modestes, la généralisation de la complémentaire santé collective peut être souhaitable pour certains salariés à condition que la subvention offerte par l'employeur soit suffisamment importante pour ne pas trop imputer leur revenu disponible ${ }^{3}$. Si au contraire leur non couverture résulte d'un choix éclairé issu d'un arbitrage entre le coût et les bénéfices attendus compte tenu de leur besoin et de leurs préférences, l'introduction de l'obligation d'assurance aurait l'inconvénient de contraindre les salariés dans leur choix de consommation, réduisant ainsi leur bienêtre. Même si la non couverture semble être le plus souvent un choix contraint, certains travaux suggèrent que la décision de souscrire un contrat de couverture relève aussi d'un choix éclairé. De fait, $12 \%$ des personnes non couvertes justifient ce choix par le fait qu'elles ne souhaitent pas être assurées ou qu'elles n'en ont pas besoin et $17 \%$ le justifie par le fait qu'elles bénéficient d'une prise en charge à $100 \%$ par l'assurance maladie pour une affection de longue durée (ALD) ou une invalidité (Célant et al., 2014). Plusieurs travaux théoriques et empiriques, mais jamais menés sur données françaises, ont également mis en évidence le rôle des préférences vis-à-vis du risque sur les décisions assurantielles, les individus les moins averses au risque préférant ne pas ou être peu couverts par une assurance (Arrow, 1963 ; Hopkins et Kidd, 1996 ; Manning et Marquis, 1996 ; Barsky et al., 1997; Butler, 1999 ; Cutler et Zeckhauser, 2000 ; Monheit et Vistnes, 2006 ; Doiron et al., 2008). Enfin, un dernier motif de recours à l'assurance proposé dans la littérature serait le pré-engagement à recourir au système de soins (Grignon et Kambia-Chopin, 2009), la souscription d'un contrat correspondant ainsi à un moyen d'investir dans sa santé, comparable au recours à la prévention. Or l'une des caractéristiques de l'investissement en santé est qu'il implique un coût immédiat pour un bénéfice futur et éventuellement incertain. Dans ce cadre la demande d'assurance devrait dépendre de la préférence pour le présent,

\footnotetext{
${ }^{3}$ Voir Jusot et Wittwer (2009) et Jusot et al. (2012) pour une discussion sur ce point.
} 
c'est-à-dire de la manière dont l'individu évalue le coût subi aujourd'hui par rapport au bénéfice attendu de demain, même si à notre connaissance, aucune étude n'a exploré cette question.

Cet article propose d'étudier les effets attendus de l'ANI sur la généralisation de la complémentaire santé et d'analyser dans quelle mesure ce dispositif permettra d'améliorer l'accès à la complémentaire santé de ceux qui subissent le fait de ne pas être couverts ou conduira au contraire à contraindre ceux pour qui la non couverture est un choix. Plus précisément, il s'agira à partir de l'Enquête Santé et Protection Sociale 2012, i/ de simuler l'impact de l'introduction de l'ANI sur la proportion d'individus qui resteront non couverts par une complémentaire en population générale et selon l'état de santé, le statut socio-économique, les préférences à l'égard du temps et du risque et le motif de non couverture; ii/ d'étudier l'évolution des caractéristiques des personnes non couvertes toutes choses égales par ailleurs avant et après l'introduction de l'ANI.

Dans une première partie, nous rappelons le contexte dans lequel la généralisation de la complémentaire santé et de la portabilité ont été introduites ainsi les nouveaux décrets qui en découlent; dans une seconde partie, nous présentons les données et la méthode utilisée. Les résultats sont présentés et discutés dans une troisième puis quatrième partie.

\section{Contexte}

En Janvier 2013, l’Accord National Interprofessionnel (ANI) signé par la majorité des partenaires sociaux concernant «la compétitivité des entreprises et la sécurisation de l'emploi et des parcours professionnels des salariés » propose, en contrepartie d'une plus grande flexibilité sur le marché du travail, deux articles concernant la complémentaire santé d'entreprise. Premièrement, il s'agit de généraliser l'offre d'une complémentaire santé d'entreprise à tous les salariés du secteur privé c'est-àdire d'obliger les employeurs à mettre en place une complémentaire santé financée au moins à hauteur de $50 \%$ par l'employeur. Deuxièmement, il s'agit de généraliser et d'augmenter les droits à la portabilité, c'est-à-dire de permettre aux personnes touchant les indemnités du chômage de bénéficier plus longtemps de leur complémentaire santé d'entreprise dans les mêmes conditions que lorsqu'ils étaient salariés. La durée de ce dispositif dépend de la durée du dernier contrat de travail et ne peut excéder 12 mois.

Cet accord, voté par les parlementaires le 14 juin 2013, devra, pour la généralisation, être effectif au 1er janvier 2016; les négociations avec les organismes complémentaires devant débuter au 1er juin 2013 au niveau de la branche puis au 1er juillet 2014 au niveau des entreprises. Les entreprises qui 
n'auront pas réussi à trouver un accord au 1er juillet 2015 devront néanmoins mettre en place un contrat avec des garanties minimales ${ }^{4}$.

Plusieurs éléments de la loi du 14 juin 2013 ont fait l'objet de nombreux débats, notamment en ce qui concerne la clause de désignation ${ }^{5}$, les aides sociales et fiscales accordées aux employeurs et aux salariés $^{6}$, les garanties minimales devant être instaurées et la redéfinition des contrats responsables ${ }^{7}$ et enfin, en ce qui concerne potentiellement la non couverture, les dispenses d'affiliation à la complémentaire santé d'entreprise. Ainsi, le dernier projet de décret rendu public le 30 juin $2014^{8}$ permet à certains individus de ne pas adhérer à la complémentaire santé d'entreprise ; notamment pour les bénéficiaires de la CMU-C et de l'ACS ; les salariés et apprentis dont la durée du contrat de travail est inférieure à 12 mois sans avoir à justifier de la présence d'une complémentaire santé souscrite par ailleurs ; les salariés à temps partiel et apprentis dont le taux d'effort au contrat collectif représenterait plus de $10 \%$ de leur revenu ; les salariés déjà couverts par un contrat individuel au moment de la mise en place du contrat collectif et jusqu'à l'échéance du contrat individuel uniquement; et enfin les salariés déjà couverts par le biais de leur conjoint à un contrat collectif.

\section{Sources et méthode}

\section{L'enquête Santé et Protection Sociale}

L'étude est réalisée à partir des données de l'Enquête santé et protection sociale (ESPS) 2012, réalisée en population générale et permettant de recueillir des informations sur l'état de santé, les caractéristiques socio-économiques et l'assurance complémentaire santé des individus. Ces informations sont recueillies pour l'ensemble des membres du ménage de la personne échantillonnée dans les registres des trois grands régimes de l'Assurance maladie obligatoire (Cnamts/RSI/MSA) ${ }^{9}$. Notre échantillon se compose de 23047 individus (tableau 1).

\footnotetext{
${ }^{4}$ Initialement, le contrat devait couvrir au minimum à hauteur de $100 \%$ de la base de remboursement les consultations, les actes techniques, la pharmacie, le forfait journalier hospitalier, couvrir à hauteur de $125 \%$ de la base de remboursement les prothèses dentaires et offrir un forfait optique de $100 €$ par an.

${ }^{5}$ En ce qui concerne les négociations effectuées au niveau de la branche, la loi du 14 juin 2013 prévoyait une clause de désignation obligeant les entreprises à choisir le contrat ou l'organisme de complémentaire santé nommé dans le cadre des négociations au niveau de la branche. Jugée non conforme aux règles concurrentielles, cette clause a définitivement été retirée du texte de loi suite à la décision du Conseil Constitutionnel de décembre 2013.

${ }^{6}$ Les aides fiscales accordées aux salariés ont été restreintes à la cotisation versée par le salarié uniquement, la part employeur étant imposable au même titre le salaire.

${ }^{7}$ Les garanties minimales devant être instaurées par les entreprises correspondent à celles imposées dans le cadre de la nouvelle définition du contrat responsable devant prendre en charge la quasi-totalité des tickets modérateurs, le forfait journalier et un forfait minimal pour l'optique. Notons ici que la nouvelle définition des contrats responsables impose par ailleurs des plafonds de remboursements à hauteur de $125 \%$ (puis de $100 \%$ en 2017) pour les dépassements d'honoraires des médecins n'ayant pas signé le contrat d'accès aux soins ainsi que des plafonds de remboursements pour l'optique.

8 http://www.sante.gouv.fr/marisol-touraine-presente-5-projets-de-decrets-sur-la-generalisation-de-1-acces-a-une-couverturecomplementaire-de-qualite.html.

${ }^{9}$ Les Sections Locales Mutualistes (SLM), et donc les mutuelles d'étudiants, ne font donc pas partie de la base de sondage. Les étudiants sont interrogés dans la mesure où ils vivent dans un foyer dont le bénéficiaire sélectionné est protégé par l'un des régimes appartenant au dispositif ESPS ; mais l'enquête ne permet pas de tenir compte des ceux vivants seuls et étant assuré auprès d'un régime étudiant.
} 
L'objectif annoncé du gouvernement étant de permettre à tous les français de bénéficier d'une couverture complémentaire santé, nous retenons comme variable d'intérêt le fait d'être couvert ou non par une complémentaire santé, quel que soit son mode d'obtention et quel que soit l'âge des individus. Ainsi, en 2012, 95\% de notre échantillon est couvert par une complémentaire santé, 53\% à titre privé individuel, 35\% par une complémentaire santé collective et $6 \%$ sont bénéficiaires de la CMU-C. Parmi les 5\% de personnes non couvertes, nous retenons le motif de non couverture afin de distinguer la non couverture subie et la non couverture par choix.

Le niveau de risque de l'individu est apprécié par l'âge, le sexe et l'état de santé, mesuré par les indicateurs de santé perçue et de maladie chronique du mini-module préconisé par Eurostat. Le premier indicateur correspond à la question suivante : «Comment est votre état de santé général? » assortie des cinq modalités de réponses suivantes : «très bon ; bon ; assez bon ; mauvais ; très mauvais ». Le second correspond à la réponse à la question : «Avez-vous une maladie ou un problème de santé qui soit chronique ou de caractère durable ?», assortie des réponses « Oui ; non; je ne sais pas ». Nous retenons également le fait d'être pris en charge à $100 \%$ par la sécurité sociale pour une affection de longue durée comme indicateur du niveau de risque de l'individu mais également comme indicateur du niveau de reste à charge après remboursement par la Sécurité sociale.

Le statut sociodémographique des individus est tout d'abord mesuré par leur statut vis-à-vis de l'emploi (actif en emploi, chômeur, retraité, étudiants, personnes au foyer, autres inactifs), le type d'emploi des actifs occupés (indépendants, salariés du privé, salariés de l'Etat) et le revenu du ménage par unité de consommation. La possession d'une complémentaire santé étant très dépendant de la stabilité financière et sociale, nous utilisons un indicateur de vulnérabilité permettant d'identifier les personnes ayant vécues de grandes difficultés dans le passé : il s'agit de celles ayant répondu « oui, sans réussir à y faire face » et/ou «oui » aux deux questions suivantes : «Vous est-il déjà arrivé au cours de votre vie de rencontrer des difficultés pour payer votre loyer, vos charges ? » et «Vous est-il déjà arrivé au cours de votre vie, de souffrir durablement d'isolement à la suite d'événements subis par vous ou vos proches? ». Nous utilisons également un indicateur permettant de cibler les individus qui anticipent éventuellement de ne pas réussir à faire face en cas de difficultés financières : il s'agit de ceux ayant répondu non à la question: «En cas de difficultés, y a-t-il dans votre entourage des personnes sur qui vous puissiez compter pour vous apporter une aide matérielle?».

L'enquête ESPS présente par ailleurs l'avantage de poser deux questions permettant d'approcher les préférences des individus à l'égard du temps et du risque. La première est la suivante : «En matière d'attitude à l'égard du risque, où vous placez-vous sur une échelle de 0 à 10 ». Les individus ayant répondu 8,9 ou 10 sont considérés comme risquophiles. La deuxième question renvoie quant à elle à la préférence temporelle : «En matière d'attitude à l'égard de l'avenir, où vous placez-vous sur une échelle de 0 à $10 »$. Les individus ayant répondu 0,1 ou 2 sont considérés comme ayant une forte préférence pour le présent. 
Tableau 1 : Description de l'échantillon selon les caractéristiques socio-économiques, d'état de santé et des préférences individuelles

\begin{tabular}{|c|c|c|c|c|c|}
\hline & & & & \multirow[b]{2}{*}{ Eff. } & \multirow[b]{2}{*}{ \% pond, } \\
\hline & Eff. & $\%$ pond. & & & \\
\hline Couverture complémentaire & & & Statut vis-à-vis de l'emploi & & \\
\hline CMU-C & 1868 & 6,1 & Actifs en emploi & 9370 & 40,7 \\
\hline Contrat collectif & 8292 & 34,5 & Retraités & 4063 & 21,3 \\
\hline Contrat individuel & 11329 & 53,1 & Chômeurs & 1480 & 5,8 \\
\hline Couvert par un biais non renseigné & 196 & 0,7 & Enfants scol.; étudiants & 6573 & 25,8 \\
\hline Non couverts & 1180 & 5,0 & Femmes/Hommes au foyer & 960 & 3,9 \\
\hline Non renseigné & 182 & 0,6 & Autres inactifs & 550 & 2,4 \\
\hline \multicolumn{3}{|c|}{ Motifs de non couverture (15 ans et + non couverts) } & Non renseigné & 51 & 0,2 \\
\hline Pris en charge à $100 \%$ & 77 & 9,1 & Type d'emploi des actifs & & \\
\hline Pas les moyens, trop cher: oui & 252 & 28,2 & Salariés du privé & 6138 & 65,9 \\
\hline Ne souhaite pas être couvert: oui & 52 & 6,5 & Salariés de l'Etat & 1816 & 19,6 \\
\hline En cours d'adhésion: oui & 54 & 5,0 & Salariés d'un particulier & 355 & 3,6 \\
\hline N'y pense pas, pas le temps: oui & 44 & 4,8 & Indépendants & 893 & 9,3 \\
\hline Non renseigné & 501 & 46,3 & Autres & 168 & 1,6 \\
\hline Sexe & & & Revenu par UC & & \\
\hline Hommes & 11434 & 47,8 & Inférieur ou égal à $650 €$ & 1533 & 5,4 \\
\hline Femmes & 11612 & 52,2 & Entre $651 €$ et $1000 €$ & 3102 & 13,6 \\
\hline Age & & & Entre $1001 €$ et $1400 €$ & 4168 & 19,1 \\
\hline-18 ans & 5383 & 22,4 & Entre $1401 €$ et $2000 €$ & 4516 & 21,0 \\
\hline De 18 à 30 ans & 3760 & 14,2 & Entre $2001 €$ et $3000 €$ & 2587 & 11,8 \\
\hline De 31 à 40 ans & 2693 & 13,5 & Supérieur à $3000 €$ & 1023 & 4,8 \\
\hline De 41 à 50 ans & 3408 & 13,2 & Non renseigné & 6118 & 24,3 \\
\hline De 51 à 60 ans & 3320 & 13,3 & \multicolumn{3}{|c|}{ Soutien matériel dans l'entourage (15 ans et + ) } \\
\hline De 61 à 70 ans & 2458 & 11,2 & Oui & 9155 & 50,8 \\
\hline De 70 à 80 ans & 1271 & 6,5 & Non & 2150 & 12,3 \\
\hline+ de 80 ans & 688 & 5,6 & Oui mais n'ose pas demander & 1347 & 7,6 \\
\hline Inconnu & 66 & 0,2 & Non renseigné & 5909 & 29,3 \\
\hline Santé perçue & & & \multicolumn{3}{|c|}{ Indicateur de vulnérabilité sociale (15 ans et +) } \\
\hline Très bon & 5007 & 21,2 & Oui & 2439 & 14,3 \\
\hline Bon & 7285 & 32,5 & Non & 10327 & 57,6 \\
\hline Assez bon & 3639 & 17,4 & Non renseigné & 5795 & 28,6 \\
\hline Mauvais/très mauvais & 1005 & 5,3 & \multicolumn{3}{|l|}{ Aversion au risque (15 ans et + ) } \\
\hline Non renseigné & 6111 & 23,7 & Risquophobes & 11619 & 65,3 \\
\hline Avec une ALD & 3249 & 16,1 & Risquophiles & 1053 & 5,5 \\
\hline Sans ALD & 19647 & 83,3 & Non renseigné & 5889 & 29,2 \\
\hline Ne sait pas & 151 & 0,6 & \multicolumn{3}{|c|}{ Préférence pour le présent (15 ans et +) } \\
\hline Avec une maladie chronique & 5145 & 25,1 & Préférence pour le futur & 11002 & 60,8 \\
\hline Sans maladie chronique & 11284 & 49,1 & Préférence pourle présent & 1632 & 9,7 \\
\hline \multirow[t]{2}{*}{ Non renseigné } & 6618 & 25,8 & Non renseigné & 5927 & 29,5 \\
\hline & & & Total & 23047 & 100 \\
\hline
\end{tabular}

N.B : L'absence de réponse concernant les questions posées aux 15 ans et plus provient du non-retour de l'auto questionnaire dans lequel sont posées ces questions.

\section{Méthode d'analyse}

Cet article propose d'étudier les effets attendus de l'ANI sur la non couverture en suivant deux objectifs : (i) simuler la proportion d'individus qui resteront non couverts par une complémentaire santé en population générale et selon les caractéristiques individuelles (état de santé, statut socioéconomique, préférences à l'égard du temps et du risque, motif de non couverture); (ii) étudier l'évolution des déterminants de la non couverture toutes choses égales par ailleurs avant et après l'introduction de l'ANI. 


\section{(i) Simulation du taux de non couverture et scénarios envisagés}

Afin d'étudier l'impact de la généralisation et de la portabilité de la complémentaire santé collective sur le taux de non couverts; nous faisons l'hypothèse que, pour les populations concernées par ces dispositifs et compte tenu de leurs caractéristiques en 2012; le taux de non couverts chutera de facto à $0 \%$. Trois catégories de personnes ont été identifiées comme directement ou indirectement concernés par la généralisation et la portabilité de la complémentaire santé collective :

1/ Les salariés du secteur privé (directement concernés par la généralisation), qui sont identifiés dans l'enquête grâce au statut d'activité et au type d'emploi : il s'agit des actifs en emploi, travaillant comme salariés du secteur privé, d'un artisan, d'une association, de la sécurité sociale ou d'un particulier.

2/ Les chômeurs de courte durée (directement concernés par la portabilité), qui concernent dans notre cas les personnes anciennement salariés du secteur privé et ayant déclaré être au chômage depuis moins d'un an, l'enquête ne permettant pas de connaitre la durée exacte des droits à la portabilité. Par ailleurs, la portabilité étant effective sous condition d'indemnités au chômage, nous excluons les chômeurs ayant déclaré qu'aucune personne du foyer ne touchait des indemnités chômage ${ }^{10}$.

3/ Les ayants droit des salariés ou des chômeurs directement concernés par la généralisation et la portabilité. Sont considérés comme ayants droits les conjoints ainsi que les enfants de moins de 26 ans étudiants ou bénéficiaires du RSA.

L'identification de ces catégories nous permet d'analyser l'évolution de la non couverture selon trois scénarios : le scénario (1) évalue uniquement l'impact de la généralisation; le scénario $(1+2)$ tient compte de la généralisation et de la portabilité ; le scénario $(1+2+3)$ évalue l'impact de la généralisation et de la portabilité sous l'hypothèse que la complémentaire santé collective soit étendue aux ayants droit. Par ailleurs, dans la mesure où les projets de décret parus le 30 juin 2014 autorisent certains individus, notamment les personnes employées dans le cadre d'un CDD de moins d'1 an, à ne pas adhérer à la complémentaire santé d'entreprise, et alors même que le montant de la cotisation à un contrat collectif peut s'avérer particulièrement élevé (Jusot et al., 2012 ; Arnould et Vidal, 2008), nous testons également l'ensemble de ces scénarios sous l'hypothèse supplémentaire que les CDD de moins de 6 mois $^{11}$ refusent d'adhérer à ce dispositif.

Le taux de non couverture mesuré en 2012 et les taux simulés selon les différents scénarios sont alors utilisés pour étudier l'évolution du taux de non couverture en population générale. Sous l'hypothèse là encore que les autres caractéristiques individuelles restent inchangées, nous regardons également de quelle façon le taux de non couverture est modifié selon l'état de santé, les caractéristiques socio-

\footnotetext{
${ }^{10}$ L'enquête ne permet pas d'identifier la personne bénéficiaire d'une allocation dès lors que plusieurs personnes d'un même ménage ont déclaré être au chômage.

${ }^{11}$ La durée des CDD dans l'enquête ESPS étant uniquement identifiable en dessous de 6 mois.
} 
économiques, les préférences individuelles et parmi les non couverts en 2012, selon les motifs de non couverture.

\section{(ii) Analyse de l'évolution des déterminants de la non couverture}

Afin d'étudier dans quelle mesure les déterminants de la non couverture pourront être modifiés suite à la généralisation et la portabilité de la complémentaire santé collective, nous modélisons la probabilité d'être non couvert par une complémentaire santé en considérant successivement le statut assurantiel actuel et le statut assurantiel simulé selon les différents scénarios :

$$
\mid \begin{array}{lll}
\operatorname{Pr}\left(N C_{2012} / X, Y, Z\right)=1 & \text { si } & N C_{2012}^{*}=a+\beta_{a} X+\gamma_{a} Y+\delta_{a} Z+\varepsilon_{a}>0 \\
\operatorname{Pr}\left(N C_{(1)} / X, Y, Z\right)=1 & \text { si } & N C_{(1)}^{*}=b+\beta_{b} X+\gamma_{b} Y+\delta_{b} Z+\varepsilon_{b}>0 \\
\operatorname{Pr}\left(N C_{(1+2)} / X, Y, Z\right)=1 & \text { si } & N C_{(1+2)}^{*}=c+\beta_{c} X+\gamma_{c} Y+\delta_{c} Z+\varepsilon_{c}>0 \\
\operatorname{Pr}\left(N C_{(1+2+3)} / X, Y, Z\right)=1 & \text { si } & N C_{(1+2+3)}^{*}=d+\beta_{d} X+\gamma_{d} Y+\delta_{d} Z+\varepsilon_{d}>0
\end{array}
$$

\section{Avec:}

$N C_{2012}$ : le fait d'être non couvert en 2012

$N C_{(1)}$ : le fait d'être non couvert selon le scénario (1)

$N C_{(1+2)}:$ le fait d'être non couvert selon le scénario $(1+2)$

$N C_{(1+2+3)}$ : le fait d'être non couvert selon le scénario $(1+2+3)$

$\mathrm{X}$ : le vecteur des caractéristiques d'âge et d'état de santé (santé perçue, maladie chronique ; ALD)

Y : le vecteur des caractéristiques socio-économiques (revenu par UC, statut par rapport à l'emploi, vulnérabilité sociale, anticipation des revenus futurs)

$\mathrm{Z}$ : le vecteur des préférences à l'égard du temps et du risque (aversion au risque, préférences temporelles)

$\varepsilon_{a} ; \varepsilon_{b} ; \varepsilon_{c} ; \varepsilon_{d}$ les résidus des modèles respectifs distribués selon une loi normale et strictement orthogonaux aux vecteurs $\mathrm{X} ; \mathrm{Y} ; \mathrm{Z}$.

Sous l'hypothèse d'exogénéité de la généralisation de la complémentaire santé collective et de sa portabilité, seul le fait d'être ou de ne pas être non couvert change selon le scénario envisagé : les caractéristiques relatives aux facteurs explicatifs $(\mathrm{X}, \mathrm{Y}, \mathrm{Z})$, c'est-à-dire les effets de composition, restent quant à eux identiques. Les effets estimés $(\widehat{\beta}, \hat{\gamma}$ et $\widehat{\delta})$ sont donc directement comparables selon les scénarios envisagés puisqu'ils reflètent uniquement des écarts liés à une corrélation plus ou moins forte. Afin de tester la significativité des effets entre les scénarios, nous comparons les intervalles de confiance calculés avec une erreur à $5 \%$. 


\section{Résultats}

\section{(i) Simulation du taux de non couverts}

En simulant un taux de non couverture nul pour les populations concernées par l'ANI, nos résultats montrent que la généralisation de la complémentaire santé collective et de la portabilité permet de réduire de façon relativement modérée le taux de non couverture (tableau 2). Le taux de non couverture passe ainsi de $5 \%$ dans la configuration actuelle à $4 \%$ sous l'hypothèse de la généralisation de la complémentaire collective pour les salariés du secteur privé (scénario 1); à 3,7\% sous l'hypothèse de la généralisation de la complémentaire collective pour les salariés du secteur privé et de la portabilité pour les chômeurs de moins de 1 an (scénario 1+2) et jusqu'à 2,7 \% sous l'hypothèse la plus large qui tient également compte du bénéfice de la complémentaire collective pour des ayants droit (scénario 1+2+3). Au final, parmi l'ensemble des individus qui ne bénéficient d'aucune complémentaire santé en 2012, $80 \%$ d'entre eux resteront non couverts après la généralisation de la complémentaire santé d'entreprise (respectivement $74,4 \%$ selon le scénario incluant également la portabilité, graphique 5.1). En effet, en 2012, le taux de non couverture, qui s'élève à $5 \%$ en moyenne, est particulièrement élevé chez les chômeurs $(13,7 \%)$, les hommes et femmes au foyer $(8,9 \%)$ et les autres inactifs $(11,6 \%)$. Les actifs en emploi, directement concernés par la généralisation de la complémentaire santé d'entreprise, sont en revanche ceux pour lesquels le taux de non couverture est le plus bas $(3,7 \%)$; la proportion de non couverts atteignant $6,7 \%$ parmi les indépendants versus 4,4 parmi les salariés d'un particulier ; 3,6\% parmi les salariés du privé et 2,6\% parmi les salariés de l'Etat (graphique 1).

Tableau 2 : Taux de non couverts observé et simulés

\begin{tabular}{|c|c|c|c|}
\hline Observé & \multicolumn{3}{|c|}{ Simulé selon les groupes de populations considérés } \\
\hline $\mathbf{2 0 1 2}$ & $\mathbf{( 1 )}$ & $\mathbf{( 1 + 2 )}$ & $\mathbf{( 1 + 2 + 3 )}$ \\
\hline 5,0 & 4,0 & 3,7 & 2,7 \\
\hline
\end{tabular}

(1): Les salariés en emploi

$(1+2)$ : Les salariés en emploi + les chômeurs de courte durée anciennement salariés

$(1+2+3)$ : Les salariés en emploi + les chômeurs de courte durée anciennement salariés + leurs ayants droits 
Graphiques 1 : Taux de non couverts en 2012 selon le statut par rapport à l'emploi

1.1. Selon l'occupation principale

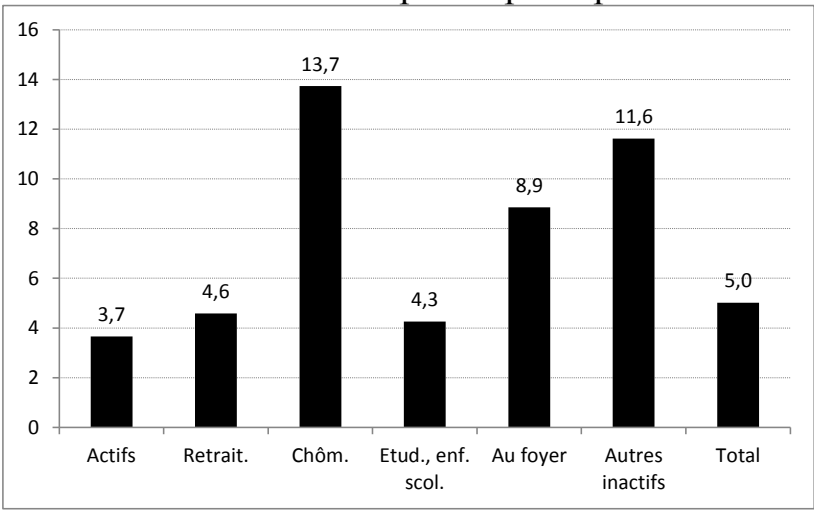

1.2. Selon le statut des actifs

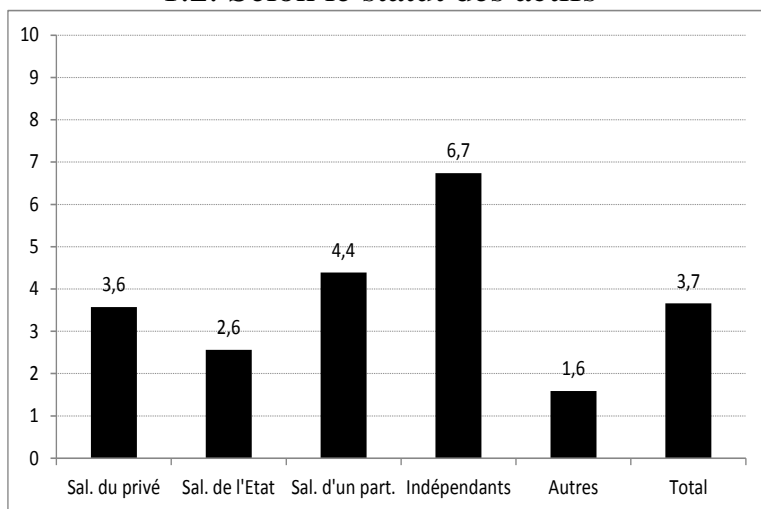

Les graphiques 2, 3 et 4 permettent d'étudier dans quelle mesure l'ANI peut modifier le taux de non couverture selon l'âge, l'état de santé, le statut socioéconomique, et les préférences individuelles ${ }^{12}$. Nous commentons tout d'abord les impacts directs de l'introduction de la généralisation de la complémentaire santé d'entreprise et de la portabilité donnés par les résultats des scénarios (1) et $(1+2)$ avant de présenter l'impact total de ce dispositif, prenant en outre en compte les effets indirects sur les ayants-droit (scénario $(1+2+3)$ ).

Concernant l'âge, la généralisation de la complémentaire santé d'entreprise et de la portabilité aura un effet particulièrement important chez les $18 / 30$ ans : leur taux de non couverture passe ainsi de $8 \%$ en 2012 à 5,6\% selon le scénario (1) et 4,5\% selon le scénario (1+2) (graphique 2.1). En s'intéressant de plus près à cette sous population (graphiques 2.2 et 2.3 ), on constate que la diminution de la non couverture concerne surtout les $18 / 20$ ans et les $21 / 24$ ans, qui sont respectivement $7,6 \%$ et $10 \%$ à être non couverts en 2012. Néanmoins, les résultats montrent également que la population des 21/24 ans restera après l'ANI, avec les populations les plus âgées, plus souvent non couverte, à hauteur de $7,2 \%$ et $6,6 \%$ selon les scénarios (1) et (1+2) pour les $21 / 24$ ans et de 6,8\% pour les plus de 80 ans. Notons par ailleurs que la diminution observée chez les jeunes de 21 à 24 ans selon les scénarios (1) et $(1+2)$ est conditionnée au fait que les CDD de moins d'un an acceptent tous d'adhérer à la complémentaire santé d'entreprise alors même que ce dispositif peut s'avérer particulièrement coûteux pour les jeunes. Ainsi, sous l'hypothèse selon laquelle les CDD de moins de 6 mois refuseraient d'y adhérer, le taux de non couverture des $21 / 24$ ans s'élèverait à 7,9\% selon le scénario (1)' et à 7,2\% selon le scénario $(1+2)$ '.

Les résultats mettent ensuite en évidence une évolution comparable du taux de non couverture selon l'état de santé des individus, que celui-ci soit apprécié par la santé perçue (graphique 2.4), le fait d'être en ALD (graphique 2.5) ou le fait d'avoir une maladie chronique (graphique 2.6). Le taux de non

\footnotetext{
${ }^{12}$ Les taux observé et simulés de non couverture sont également présentés en annexe sous forme de tableau.
} 
couverture diminuera sensiblement de -1 point dans chaque sous population; et ce de manière très proche selon les scénarios (1) et (1+2). Par exemple, le taux de non couverture, qui s'élève à 4,8\% pour les individus ayant déclaré une «assez bonne santé » est évalué à 3,9\% dans le cas du scénario (1) et à 3,7\% dans le cas du scénario $(1+2)$. Là encore cependant, nous montrons que le taux de non couverture restera particulièrement élevé parmi les individus qui se déclarant en mauvaise santé $(8,8 \%$ selon les scénarios (1) et (1+2)) ainsi que chez ceux en ALD (5,8\%).

Concernant le statut vis-à-vis de l'emploi (graphique 3.1), le taux de non couverture diminuera, de manière attendue, particulièrement parmi les actifs selon le scénario (1) et parmi les chômeurs selon le scénario $(1+2)$. Cependant, le taux de non couverture restera relativement très élevé pour les chômeurs : 9,1 \% selon le scénario (1+2) alors que celui des actifs, qui était déjà le plus bas en 2012, est estimé à $1,2 \%$ selon les scénarios (1) et $(1+2)$. De même, l'analyse du taux de non couverture selon le revenu (graphique 3.2) montre que celui-ci restera fortement décroissant avec le niveau de revenu après la généralisation de la complémentaire santé collective et la portabilité. Ainsi, dans le scénario $(1+2)$, la proportion de personnes sans complémentaire santé est estimée à $12,7 \%$ pour les individus dont le revenu par UC est inférieur à $650 €$ par mois, versus $0,7 \%$ pour ceux dont le revenu par UC se situe entre $2001 €$ et $3000 €$ et $2,9 \%$ pour ceux dont le revenu par UC se situe au-dessus de $3000 €$. Les individus en situation de vulnérabilité sociale sont également ceux pour lesquels le taux de non couverture restera le plus élevé $(6,9 \%$ selon le scénario 1 et $6,3 \%$ selon le scénario $(1+2)$, graphique 3.3). Toutefois, la diminution du taux de non couverture sera plus marqué parmi les personnes vulnérables (-2 points) que parmi les personnes non vulnérables $(-0,8)$; ce qui souligne la situation de précarité sociale de certains salariés en emploi. Il en est de même parmi ceux qui pensent ne pas pouvoir bénéficier d'un soutien matériel en cas de difficulté (graphique 3.4). Néanmoins, malgré une baisse de -1,6 points du taux de non couverts selon le scénario (1) parmi les personne ne disposant pas de soutien matériel (versus -1,1 point parmi les personnes disposant de soutien matériel); ces derniers resteront néanmoins non couverts à hauteur de 5,7\% selon le scénario (1) et 5,4\% selon le scénario $(1+2)$.

Concernant les préférences individuelles, les résultats montrent que même si les personnes les plus risquophiles resteront plus fréquemment non couvertes que les personnes averses au risque, la diminution du taux de non couverture est plus élevée parmi les individus les plus risquophiles, c'est-àdire ceux qui la non couverture est potentiellement choisie et non subie : le taux de non couverture diminue ainsi de $-3,1$ points selon le scénario (1) parmi les risquophiles versus - 0,9 points parmi les risquophobes (graphique 4.1). De même, les personnes ayant une forte préférence pour le présent resteront plus fréquemment non couvertes que les personnes ayant une plus forte préférence pour le futur mais la diminution du taux de non couverture est plus élevée parmi les individus ayant une forte préférence pour le présent (-1,6 points versus -1 point parmi les personnes ayant une préférence pour le futur). 
Ainsi, si l'on restreint l'analyse aux individus non couverts en 2012 et que l'on estime l'impact de l'ANI sur le taux de non couverture selon les motifs de non couverture, nous mettons en évidence une diminution du taux de non couverture beaucoup plus importante parmi ceux pour qui la non couverture est choisie plutôt que subie. En effet, parmi les personnes ayant déclaré qu'elles souhaiteraient être couvertes mais qu'elles n'en n'ont pas les moyens, respectivement $80,7 \%$ et $74,1 \%$ resteront non couvertes selon les scénarios (1) et $(1+2)$ alors que le taux de non couverture passera de $100 \%$ à $64 \%$ et $62,2 \%$ selon les scénarios (1) et (1+2) parmi celles ayant déclaré qu'elles ne souhaitaient pas être couvertes. L'ANI devrait enfin réduire la non couverture frictionnelle puisque le taux de non couverture serait réduit à respectivement $62,2 \%$ et $54,1 \%$ selon le scénario (1) et $(1+2)$ chez les personnes ayant déclaré être en cours d'adhésion.

Si l'on considère en revanche les effets indirects de l'ANI sur les ayants-droit potentiels (enfants et conjoints) selon le scénario $(1+2+3)$, les résultats montrent que le taux de non couverture diminuera sensiblement chez les moins de 30 ans, de sorte que les populations les plus âgées seront les seules à être principalement concernées par la non couverture (graphiques 2.1 et 2.2). Selon ce scénario, le taux de non couverture diminuerait également sensiblement plus parmi les individus en très bonne santé perçue (-1,5 points versus -0.7 point parmi ceux en mauvaise santé ; graphique 2.4) et ceux sans ALD (-1,2 points versus -0.4 point parmi ceux avec une ADL; graphique 2.5$)$. Il y aurait également une diminution de la non couverture parmi les inactifs (graphique 3.1) et les plus précaires (graphique 3.2), bien que celle-ci restera toujours plus élevée dans ces deux sous-populations. Au final, si l'on considère l'ensemble des personnes sans complémentaire santé en 2012, le taux de non couverture serait évalué à $53,5 \%$ selon le scénario $(1+2+3)$ versus $79,7 \%$ et $74,4 \%$ selon les scénarios (1) et $(1+2)$, révélant ainsi l'importance des ménages sans aucune complémentaire santé. 
Graphiques 2 : Taux de non couverts selon l'âge et l'état de santé

2.1. Selon l'âge et sur l'ensemble de la population

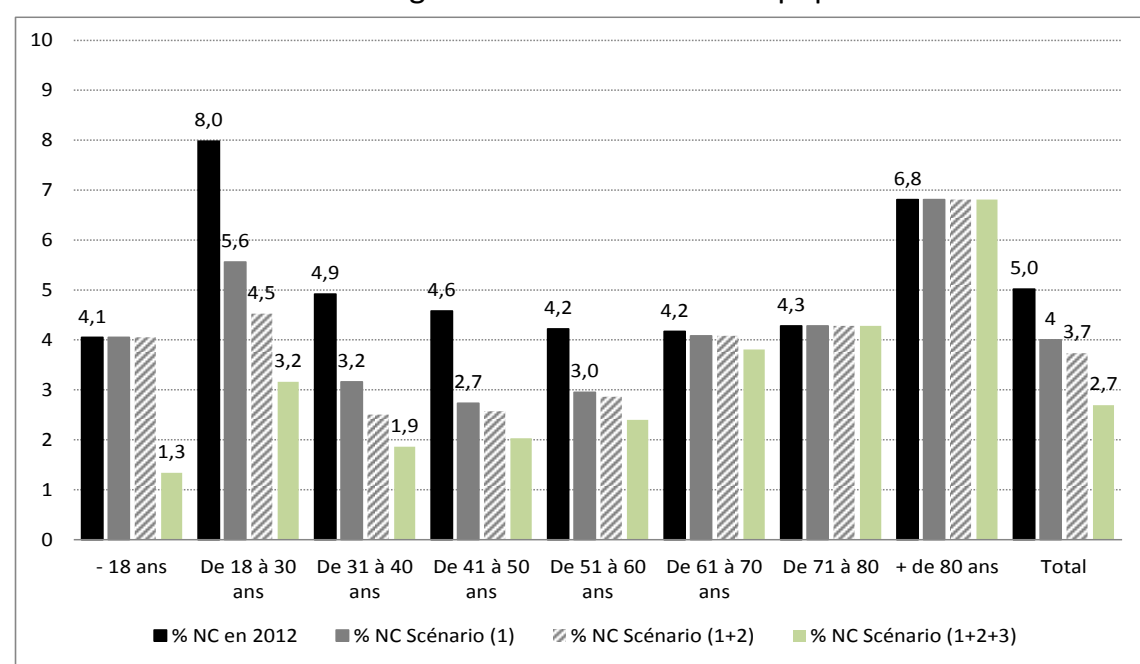

2.3. Selon l'âge, en ciblant les moins de 30 ans et sous l'hypothèse que les CDD de moins de 6 mois refusent d'adhérer à la CSE

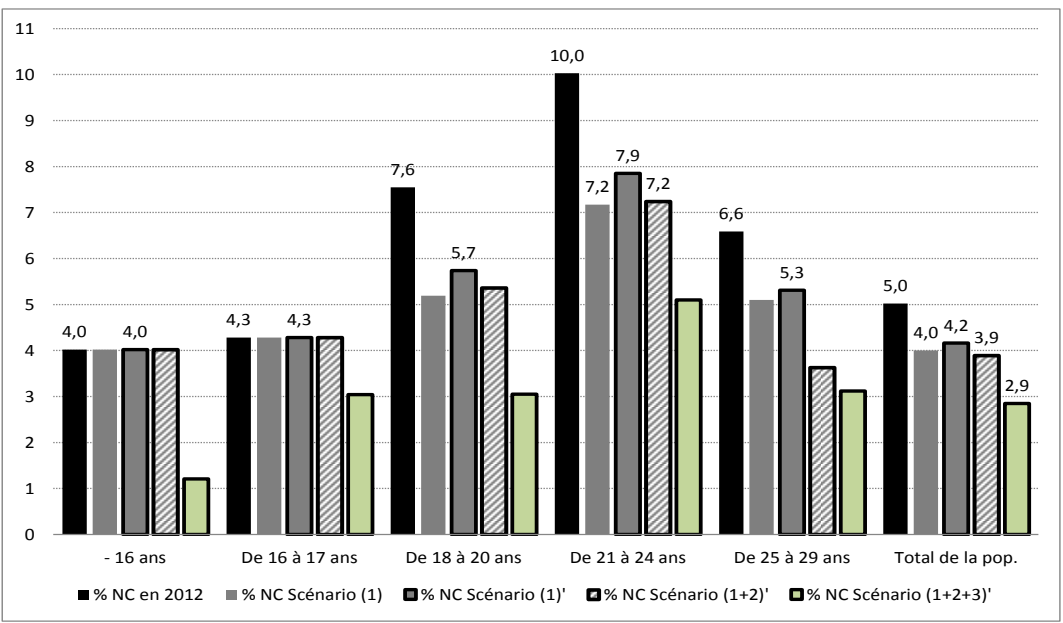

2.2. Selon l'âge, en ciblant les moins de 30 ans

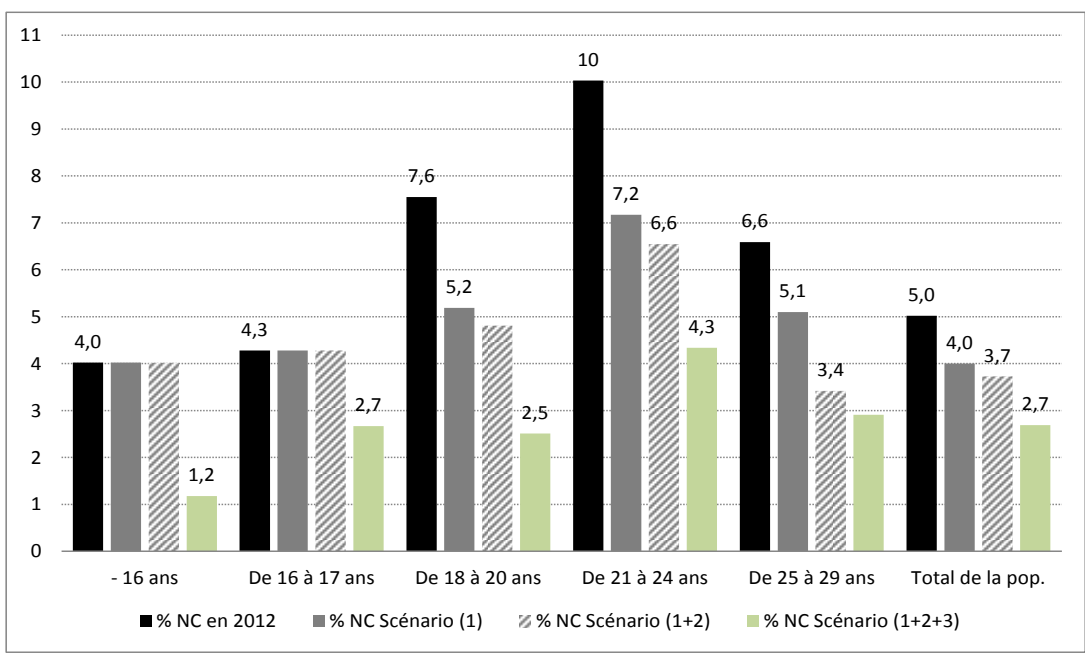

2.4. Selon la santé perçue

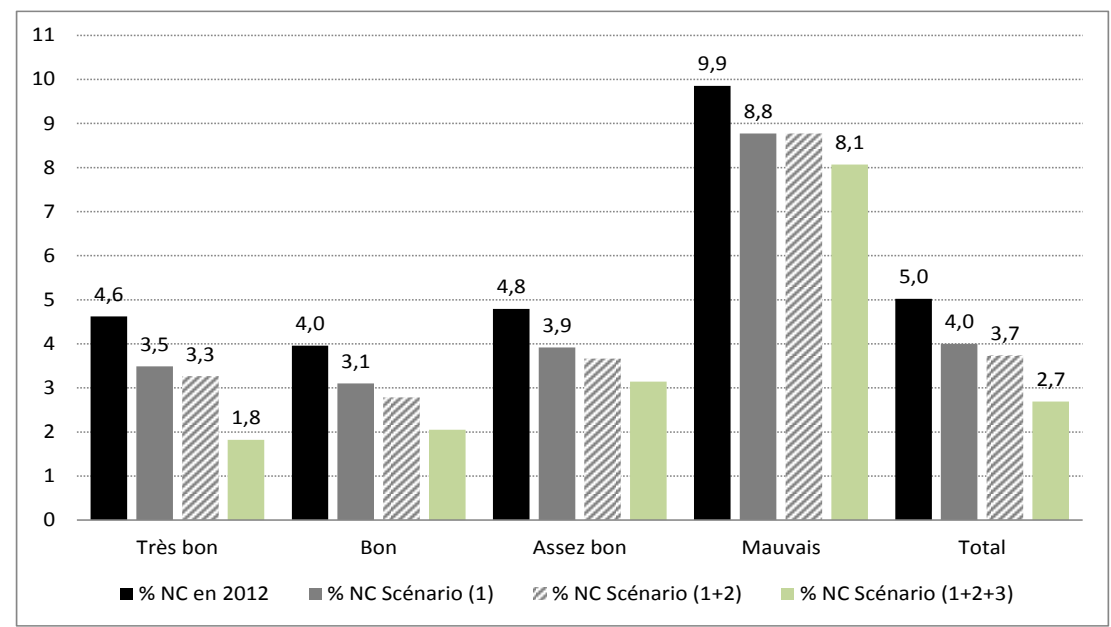


2.5. Selon le fait d'être en ALD

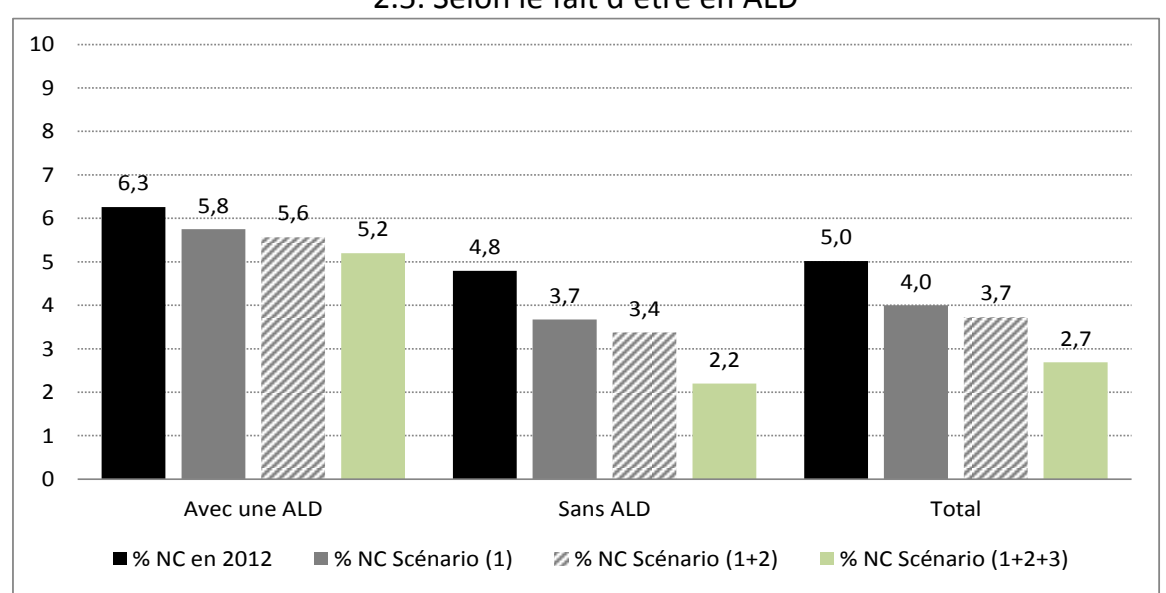

2.6. Selon le fait d'avoir une maladie chronique

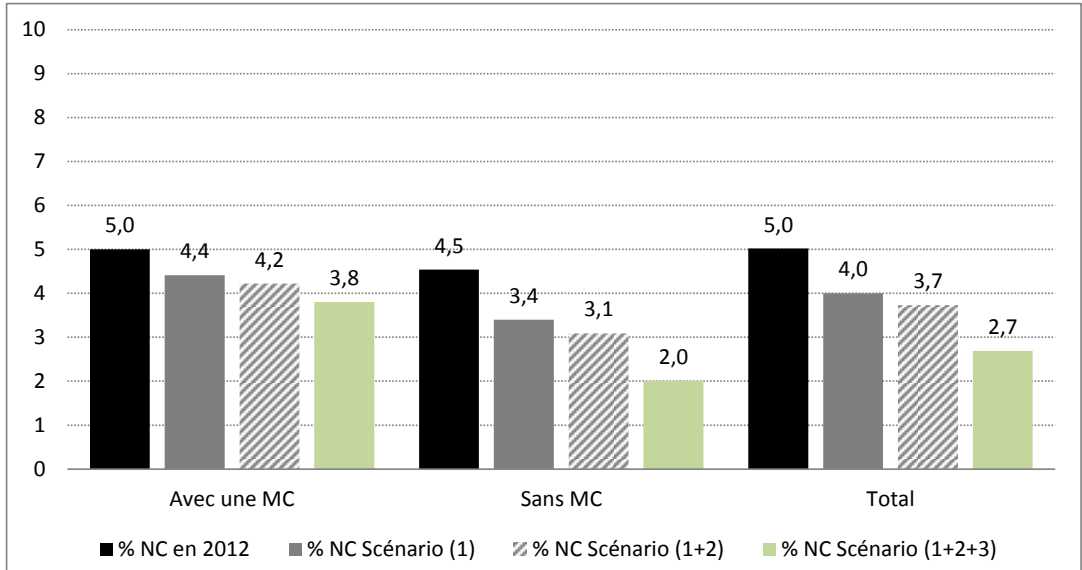

\section{Graphiques 3 : Taux de non couverts selon les caractéristiques socio-économiques}
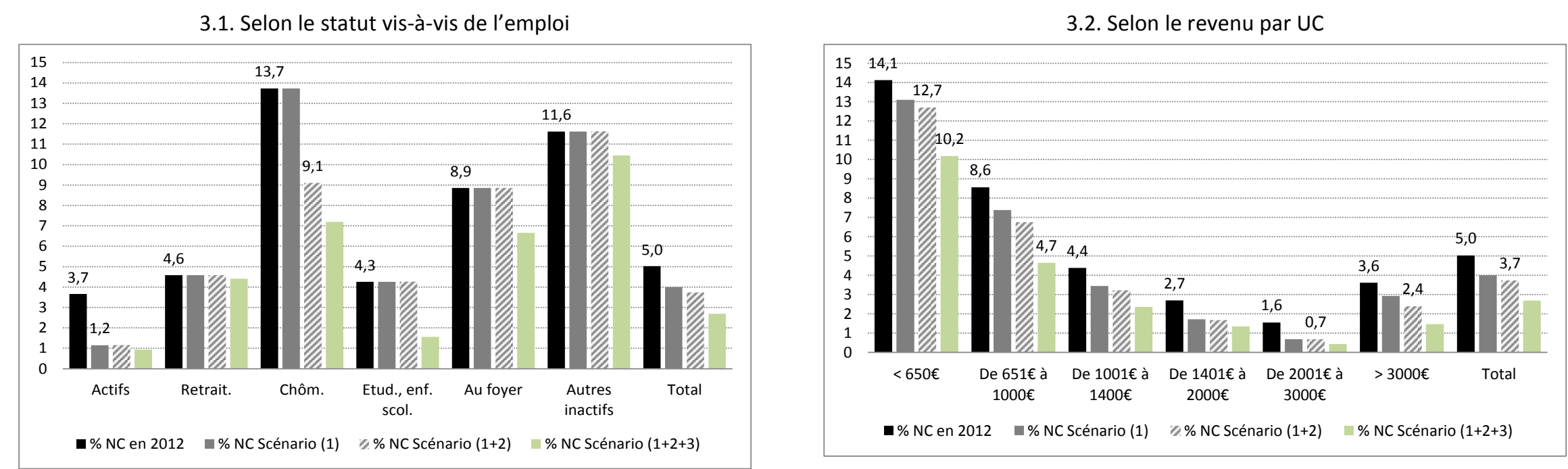
3.3. Selon l'indicateur de vulnérabilité sociale ( 15 ans et + )

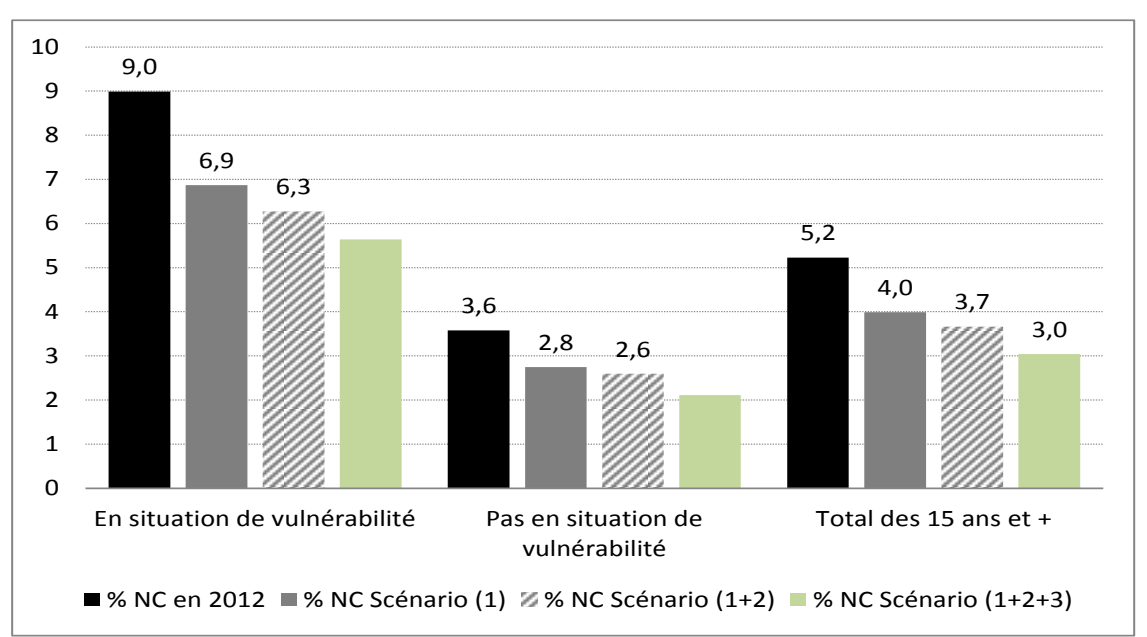

3.4. Selon l'existence d'un soutien matériel (15 ans et + )

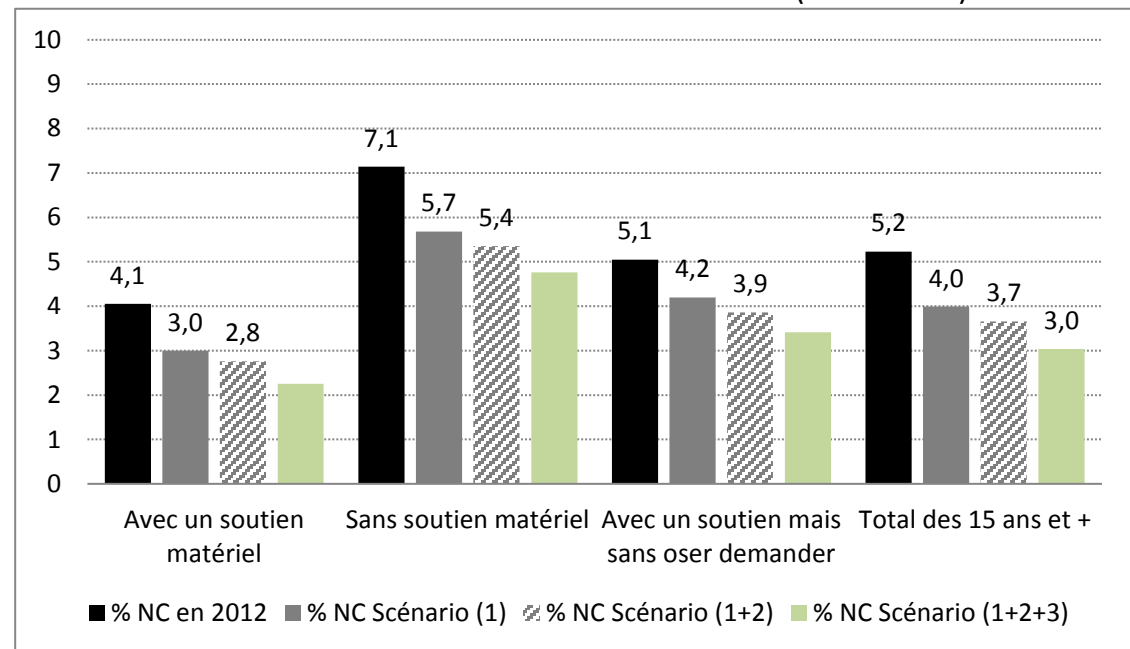

Graphiques 4 : Taux de non couverts selon les préférences à l'égard du temps et du risque

4.1. Selon l'aversion au risque ( 15 ans et + )

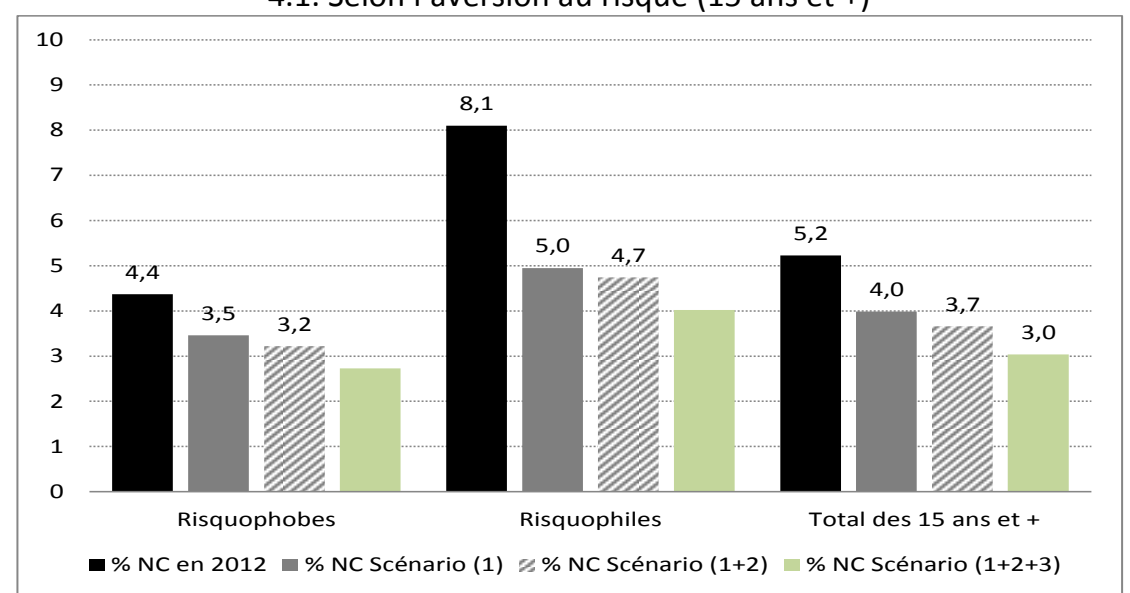

4.2. Selon la préférence temporelle ( 15 ans et + )

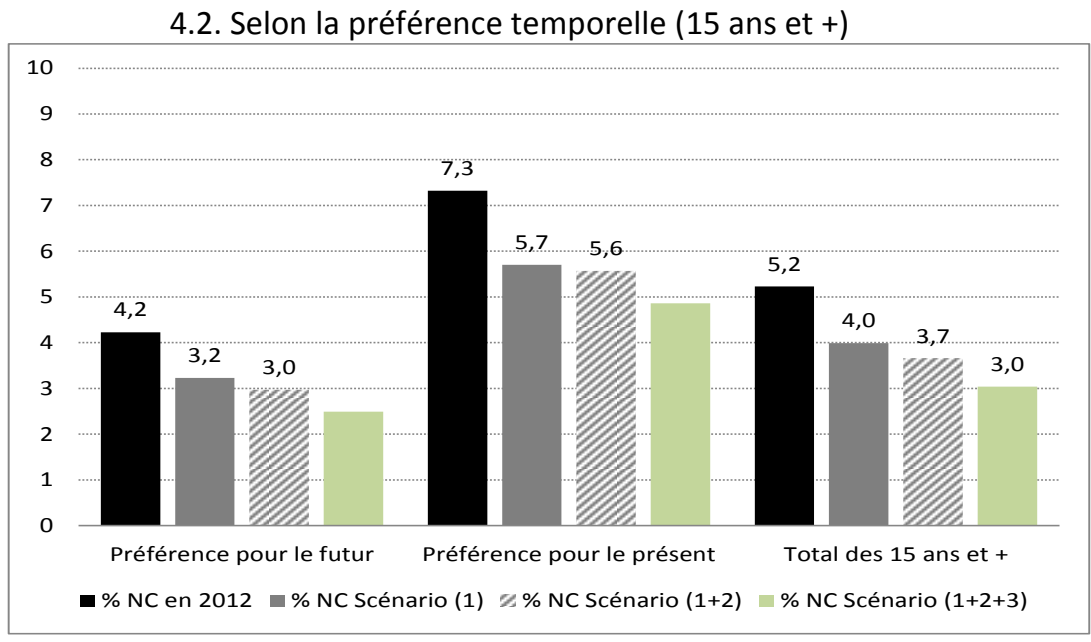


Graphiques 5 : Taux de non couverts simulés parmi les non couverts en 2012 et selon les motifs de non couverture

Graphique 5.1 : Parmi les non couverts en 2012

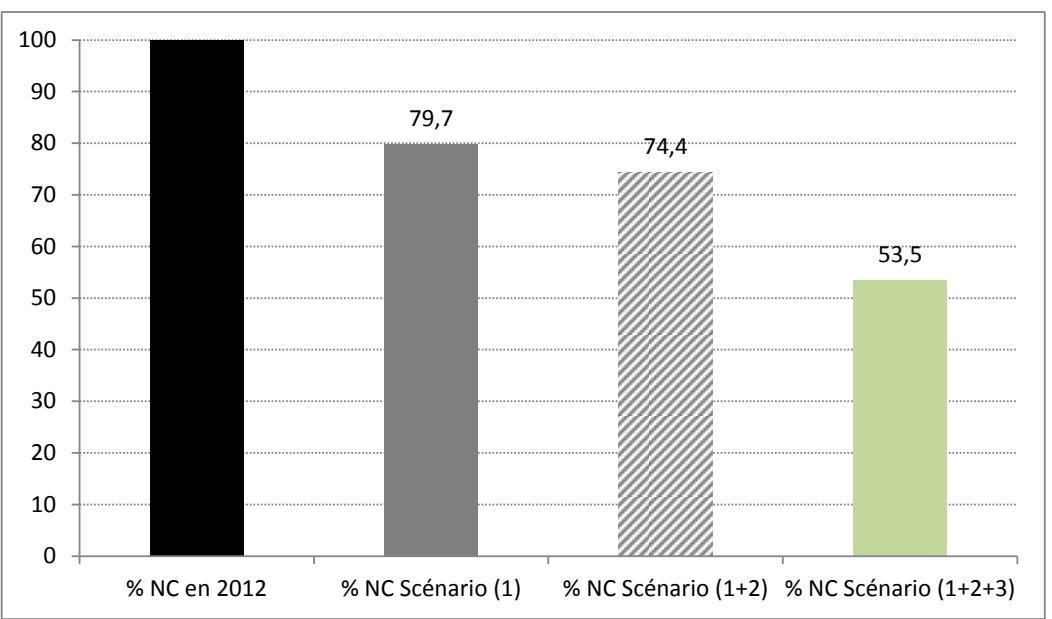

Graphique 5.2 : Selon les motifs de non couverture (15 ans et + )

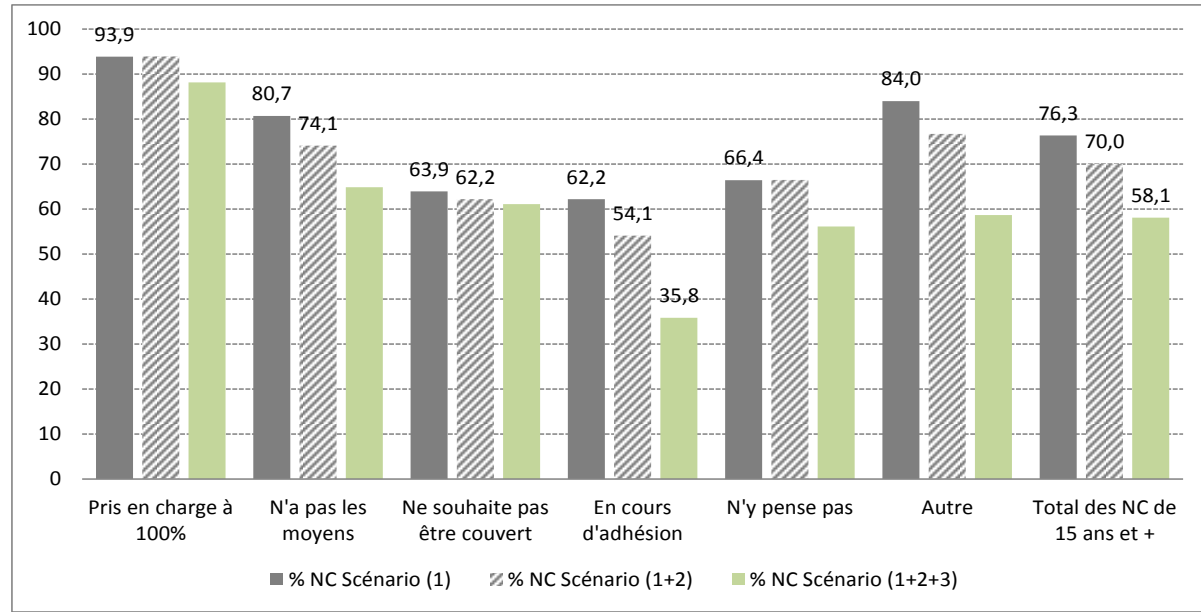




\section{(ii) Les déterminants de la non couverture}

Les résultats de la modélisation de la non couverture en 2012 et selon les scénarios $(1),(1+2)$ et $(1+2+3)$ sont présentés dans le tableau 3. Pour une meilleure lisibilité, nous présentons les principaux résultats sous formes graphiques; le chevauchement des intervalles de confiance indiquant que les effets ne sont pas significativement selon les différents scénarios envisagés.

En 2012, nous montrons que toutes choses égales par ailleurs, la probabilité de ne pas être couvert par une complémentaire santé est plus élevée chez les hommes (+1,2 points), les jeunes âgés de 18 à 30 ans $(+4,2$ points comparativement à ceux âgés entre 51 et 60 ans) ainsi que ceux de 80 ans et plus $(+2,8$ points). De manière cohérente avec les travaux déjà réalisés sur la demande de complémentaire santé, les variables d'état de santé sont assez peu significatives : seul le fait de déclarer un mauvais état de santé perçu plutôt qu'un très bon état de santé est corrélé positivement avec un accroissement de 2.2 points du taux de non couverture. Notre modèle confirme ensuite le rôle prépondérant des variables socio-économiques. Ainsi, plus le revenu par UC est faible, plus la probabilité de ne pas être couvert par une complémentaire santé est élevée : $+2,5$ points pour ceux dont le revenu par UC se situe entre $1201 €$ et $1400 € ;+7,1$ lorsque le revenu par UC se situe entre $651 €$ et $1200 €$ et $+10,1$ points lorsqu'il est inférieur à $650 €$ par mois, qui correspond pourtant au seuil d'éligibilité à la CMUC. Les individus en situation de vulnérabilité sociale sont également plus souvent concernés $(+3,2$ points). Nous montrons enfin que les individus ayant une forte préférence pour le présent ainsi que ceux ayant une certaine attirance à l'égard du risque sont également plus souvent non couverts : respectivement $+1,1$ points et $+1,8$ points.

La comparaison des résultats de la modélisation de la non couverture en 2012 et selon les scénarios $(1),(1+2)$ et $(1+2+3)$ suggère que l'ANI va sensiblement modifier les caractéristiques des personnes non couvertes. Même si, comme précédemment montré et comparativement aux personnes âgées de 51 à 60 ans, la diminution du taux de non couverture touche particulièrement les 18/30 ans, la probabilité d'être non couvert restera significativement plus élevée après l'ANI dans cette tranche d'âge. La diminution de l'association entre cette tranche d'âge et la non couverture entre la situation en 2012 et les scénarios (1) et (1+2) est à la limite de la significativité (graphique 6.2), à condition néanmoins que les CDD de moins de 6 mois ne refusent pas d'adhérer à la complémentaire santé d'entreprise (graphique 6.3). En effet, seul le scénario $(1+2+3)$ permet de réduire significativement et sans condition le lien entre la non couverture et le fait d'être âgé de 18 à 30 ans comparativement aux personnes âgées de 51 à 60 ans. Les 80 ans en revanche resteront toujours plus fréquemment non couverts que les 51/60 ans sans que l'on note une différence significative de modification d'association entre cette tranche d'âge et le taux de non couverture selon le scénario envisagé (graphique 6.4). 
L'ANI modifiera peu l'association entre l'état de santé et la non couverture. Ainsi la mauvaise santé perçue reste significativement associée au fait de ne pas être couvert après la généralisation de la couverture complémentaire d'entreprise (scénarios (1)) et de la portabilité (scénario $(1+2)$ ) et l'on ne note pas de différence significative dans l'ampleur de cette association selon ces deux scénarios (graphique 6.1). Notons toutefois que dans le scénario $(1+2+3)$, la non couverture n'est plus significativement reliée à la mauvaise santé perçue.

En revanche, nos résultats montrent un renforcement très marqué du lien entre le statut vis-à-vis de l'emploi et le fait d'être non couvert. Ainsi, alors même que les chômeurs sont directement concernés en partie par le scénario $(1+2)$, nos résultats montrent un renforcement significatif du lien avec le fait de ne pas être couverts (graphique 7.2). En effet, le fait d'être chômeur accroit de 4.1 points la probabilité d'être non couvert par rapport au fait d'être actif en emploi en 2012 versus un accroissement de 11,1 points selon le scénario (1) et de 7,1 points selon le scénario $(1+2)$. Il en est de même pour les retraités, les étudiants, les personnes au foyer et les autres inactifs (graphiques 7.1, 7.3 et 7.4). Seul le troisième scénario, qui tient généralise l'accès à la complémentaire santé d'entreprise aux ayants-droit, permet de conserver le même lien que celui observé en 2012. L'effet du revenu n'est quant à lui pas significativement différent selon les différents scénarios envisagés (graphique 7.5) : les plus pauvres seront toujours ceux seront le plus souvent non couverts par une complémentaire santé. Néanmoins, avec l'association entre la vulnérabilité sociale et la non couverture diminue légèrement après l'ANI, bien qu'il reste significativement différent de 0 (graphique 7.6).

Enfin, il est intéressant de noter que, les préférences des individus à l'égard du risque et du temps ne sont plus, après l'introduction de l'ANI, significativement associées au fait de ne pas être couvert par une complémentaire santé. 
Tableau 3 : Modélisation de la non couverture en 2012 et selon les scénarios (1), (1+2) et $(1+2+3)$

\begin{tabular}{|c|c|c|c|c|c|c|c|c|c|c|c|c|c|c|c|c|}
\hline & \multicolumn{4}{|c|}{ Pr.(NC=1) en 2012} & \multicolumn{4}{|c|}{$\operatorname{Pr}(\mathrm{NC}=1$ Scén. 1) } & \multicolumn{4}{|c|}{$\operatorname{Pr}(N C=1$ Scén. $(1+2))$} & \multicolumn{4}{|c|}{$\operatorname{Pr}(\mathrm{NC}=1$ Scén. $(1+2+3))$} \\
\hline & EM & Pr. & IC - & IC + & EM & Pr. & IC - & IC + & EM & Pr. & IC - & IC+ & EM & Pr. & IC - & IC + \\
\hline \multicolumn{17}{|l|}{ Sexe - Ref: Hommes } \\
\hline Femmes & $-1,2$ & 0,000 & $-1,7$ & $-0,7$ & $-0,8$ & 0,000 & $-1,2$ & $-0,4$ & $-0,7$ & 0,000 & $-1,1$ & $-0,3$ & $-0,7$ & 0,000 & $-1,0$ & $-0,4$ \\
\hline \multicolumn{17}{|l|}{ Age - Ref: $51-60$ ans } \\
\hline Moins de 18 ans & 1,3 & 0,113 & $-0,4$ & 3,0 & 0,3 & 0,622 & $-0,9$ & 1,5 & 0,1 & 0,913 & $-1,1$ & 1,2 & $-0,5$ & 0,229 & $-1,3$ & 0,3 \\
\hline $18 / 30$ ans & 4,2 & 0,000 & 2,7 & 5,8 & 2,1 & 0,000 & 0,9 & 3,3 & 1,6 & 0,002 & 0,4 & 2,7 & 0,8 & 0,020 & 0,0 & 1,6 \\
\hline $31 / 40$ ans & 1,2 & 0,044 & $-0,1$ & 2,4 & 0,7 & 0,154 & $-0,3$ & 1,7 & 0,2 & 0,624 & $-0,7$ & 1,2 & 0,0 & 0,991 & $-0,7$ & 0,7 \\
\hline $41 / 50$ ans & 0,4 & 0,497 & $-0,7$ & 1,4 & 0,2 & 0,677 & $-0,7$ & 1,1 & 0,1 & 0,800 & $-0,7$ & 1,0 & $-0,1$ & 0,733 & $-0,7$ & 0,5 \\
\hline $61 / 70$ ans & 0,3 & 0,677 & $-1,3$ & 2,0 & 0,3 & 0,667 & $-1,0$ & 1,5 & 0,2 & 0,716 & $-1,0$ & 1,4 & 0,7 & 0,140 & $-0,3$ & 1,7 \\
\hline $71 / 80$ ans & 0,8 & 0,433 & $-1,3$ & 2,8 & 0,5 & 0,465 & $-1,0$ & 2,0 & 0,4 & 0,546 & $-1,0$ & 1,8 & 1,1 & 0,040 & $-0,2$ & 2,5 \\
\hline Plus de 80 ans & 2,8 & 0,018 & 0,0 & 5,5 & 2,0 & 0,021 & $-0,1$ & 4,0 & 1,8 & 0,031 & $-0,2$ & 3,7 & 2,5 & 0,000 & 0,6 & 4,5 \\
\hline \multicolumn{17}{|l|}{ En ALD - Ref: Non } \\
\hline Oui & 0,3 & 0,501 & $-0,6$ & 1,2 & 0,2 & 0,458 & $-0,4$ & 0,9 & 0,3 & 0,432 & $-0,4$ & 0,9 & 0,2 & 0,324 & $-0,3$ & 0,7 \\
\hline Non renseigné & $-1,5$ & 0,289 & $-3,7$ & 0,8 & $-0,6$ & 0,608 & $-2,5$ & 1,4 & $-0,3$ & 0,757 & $-2,4$ & 1,7 & 0,4 & 0,664 & $-1,5$ & 2,3 \\
\hline \multicolumn{17}{|l|}{ Maladie Chronique - Ref: Non } \\
\hline Oui & $-0,8$ & 0,052 & $-1,6$ & 0,0 & $-0,4$ & 0,198 & $-1,0$ & 0,2 & $-0,4$ & 0,222 & $-1,0$ & 0,2 & $-0,1$ & 0,675 & $-0,6$ & 0,4 \\
\hline Non renseigné & 0,4 & 0,607 & $-1,2$ & 2,0 & 0,5 & 0,445 & $-0,7$ & 1,7 & 0,6 & 0,295 & $-0,6$ & 1,8 & 0,5 & 0,278 & $-0,4$ & 1,4 \\
\hline \multicolumn{17}{|l|}{ Santé perçue - Ref: Très bonne } \\
\hline Assez bonne & 0,2 & 0,695 & $-0,7$ & 1,0 & 0,0 & 0,888 & $-0,6$ & 0,7 & 0,0 & 0,975 & $-0,6$ & 0,6 & $-0,1$ & 0,687 & $-0,5$ & 0,4 \\
\hline Mauvaise et très mauvaise & 2,2 & 0,004 & 0,5 & 4,0 & 1,3 & 0,017 & 0,1 & 2,6 & 1,4 & 0,011 & 0,1 & 2,7 & 0,7 & 0,074 & $-0,2$ & 1,6 \\
\hline Non renseigné & $-0,1$ & 0,913 & $-1,8$ & 1,6 & $-0,3$ & 0,673 & $-1,4$ & 0,9 & $-0,3$ & 0,576 & $-1,5$ & 0,8 & $-0,1$ & 0,866 & $-1,0$ & 0,8 \\
\hline \multicolumn{17}{|c|}{ Statut par rapport à l'emploi - Ref: Actifs occupés } \\
\hline Retraités & 1,5 & 0,080 & $-0,3$ & 3,3 & 5,5 & 0,000 & 3,1 & 7,9 & 5,1 & 0,000 & 2,8 & 7,3 & 2,7 & 0,000 & 1,1 & 4,2 \\
\hline Chômeurs & 4,1 & 0,000 & 2,6 & 5,6 & 11,1 & 0,000 & 8,8 & 13,4 & 7,1 & 0,000 & 5,2 & 9,0 & 4,1 & 0,000 & 2,6 & 5,5 \\
\hline Etudiants & $-0,8$ & 0,180 & $-1,9$ & 0,3 & 3,4 & 0,000 & 2,0 & 4,8 & 3,5 & 0,000 & 2,1 & 4,9 & 1,1 & 0,009 & 0,2 & 2,0 \\
\hline Au foyer & 3,2 & 0,000 & 1,4 & 5,1 & 9,2 & 0,000 & 6,4 & 11,9 & 8,6 & 0,000 & 6,0 & 11,2 & 4,7 & 0,000 & 2,8 & 6,6 \\
\hline Autres inactifs & 4,5 & 0,000 & 2,0 & 7,1 & 11,3 & 0,000 & 7,6 & 15,0 & 10,5 & 0,000 & 7,0 & 14,0 & 7,4 & 0,000 & 4,6 & 10,3 \\
\hline \multicolumn{17}{|c|}{ Revenu par UC - Ref: Supérieur à $2000 €$} \\
\hline Inférieur à $650 €$ & 10,1 & 0,000 & 7,4 & 12,9 & 8,0 & 0,000 & 5,5 & 10,5 & 8,9 & 0,000 & 6,2 & 11,6 & 8,1 & 0,000 & 5,3 & 11,0 \\
\hline Entre $651 €$ et $1200 €$ & 7,1 & 0,000 & 5,1 & 9,1 & 5,4 & 0,000 & 3,6 & 7,2 & 5,6 & 0,000 & 3,8 & 7,5 & 3,8 & 0,000 & 2,2 & 5,4 \\
\hline Entre $1201 €$ et $1400 €$ & 2,5 & 0,000 & 1,1 & 3,9 & 2,2 & 0,000 & 1,0 & 3,5 & 2,4 & 0,000 & 1,1 & 3,6 & 2,0 & 0,000 & 0,9 & 3,2 \\
\hline Entre $1401 €$ et $2000 €$ & 0,6 & 0,273 & $-0,5$ & 1,8 & 0,4 & 0,446 & $-0,6$ & 1,4 & 0,5 & 0,273 & $-0,5$ & 1,6 & 0,7 & 0,100 & $-0,2$ & 1,6 \\
\hline Non renseigné & 3,4 & 0,000 & 2,1 & 4,8 & 2,9 & 0,000 & 1,7 & 4,1 & 3,0 & 0,000 & 1,8 & 4,2 & 2,1 & 0,000 & 1,0 & 3,1 \\
\hline \multicolumn{17}{|l|}{ Vulnérabilité sociale - Ref: Absence } \\
\hline En vulnérabilité & 3,2 & 0,000 & 2,0 & 4,4 & 1,7 & 0,000 & 0,8 & 2,6 & 1,5 & 0,000 & 0,7 & 2,4 & 1,2 & 0,000 & 0,5 & 1,8 \\
\hline Non renseigné & 1,7 & 0,099 & $-0,4$ & 3,7 & 1,1 & 0,118 & $-0,3$ & 2,6 & 1,1 & 0,131 & $-0,4$ & 2,5 & 0,6 & 0,224 & $-0,4$ & 1,7 \\
\hline \multicolumn{17}{|c|}{ Aide des proches en cas de diff. fin. - Ref: Oui } \\
\hline Non & 1,0 & 0,039 & 0,0 & 2,1 & 0,7 & 0,066 & $-0,1$ & 1,5 & 0,6 & 0,090 & $-0,2$ & 1,4 & 0,4 & 0,174 & $-0,2$ & 0,9 \\
\hline Oui mais n'ose pas demander & 0,7 & 0,232 & $-0,5$ & 2,0 & 0,6 & 0,172 & $-0,4$ & 1,6 & 0,5 & 0,231 & $-0,4$ & 1,5 & 0,4 & 0,219 & $-0,3$ & 1,1 \\
\hline Non renseigné & 0,2 & 0,826 & $-1,7$ & 2,1 & 0,6 & 0,415 & $-0,8$ & 2,0 & 0,5 & 0,451 & $-0,9$ & 1,9 & 0,4 & 0,379 & $-0,6$ & 1,4 \\
\hline \multicolumn{17}{|l|}{ Préférence pour le présent - Ref: Non } \\
\hline Oui & 1,1 & 0,032 & 0,0 & 2,2 & 0,5 & 0,187 & $-0,3$ & 1,3 & 0,6 & 0,132 & $-0,2$ & 1,4 & 0,3 & 0,290 & $-0,3$ & 0,9 \\
\hline Non renseigné & 0,8 & 0,628 & $-2,6$ & 4,3 & 0,0 & 0,977 & $-2,5$ & 2,6 & 0,0 & 0,987 & $-2,5$ & 2,5 & 0,4 & 0,644 & $-1,4$ & 2,2 \\
\hline \multicolumn{17}{|l|}{ Risquophile - Ref: Non } \\
\hline Oui & 1,8 & 0,007 & 0,3 & 3,2 & 0,4 & 0,478 & $-0,7$ & 1,4 & 0,5 & 0,298 & $-0,5$ & 1,6 & 0,7 & 0,058 & $-0,1$ & 1,6 \\
\hline Non renseigné & $-0,2$ & 0,893 & $-3,7$ & 3,2 & $-0,2$ & 0,883 & $-2,8$ & 2,4 & $-0,3$ & 0,829 & $-2,8$ & 2,2 & $-0,7$ & 0,412 & $-2,5$ & 1,0 \\
\hline $\operatorname{Pr}(\mathrm{Y}=1 / \mathrm{X}=$ moyenne $)$ & \multicolumn{4}{|c|}{3,99} & \multicolumn{4}{|c|}{2,54} & & 2,4 & 47 & & & 1,5 & 54 & \\
\hline
\end{tabular}

EM : Effet marginal calculé en points

Pr. Probabilité que l'effet marginal soit nul

IC : Intervalles de confiance calculés avec une erreur totale de $5 \%$ autour de de l'effet marginal (respectivement 2,5\% à gauche et à droite). 
Graphiques 6 : Lien entre la non couverture et les variables d'âge et d'état de santé

6.1. Mauvaise santé perçue (Ref : bonne santé)

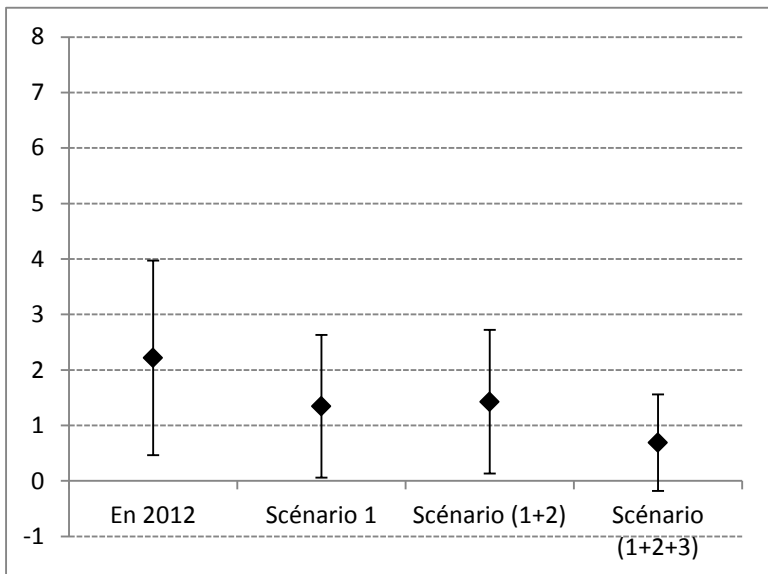

6.3 Entre 18 et 30 ans (Ref : bonne santé) et selon l'hypothèse selon laquelle les CDD de moins de 6 refusent d'adhérer à la CSE

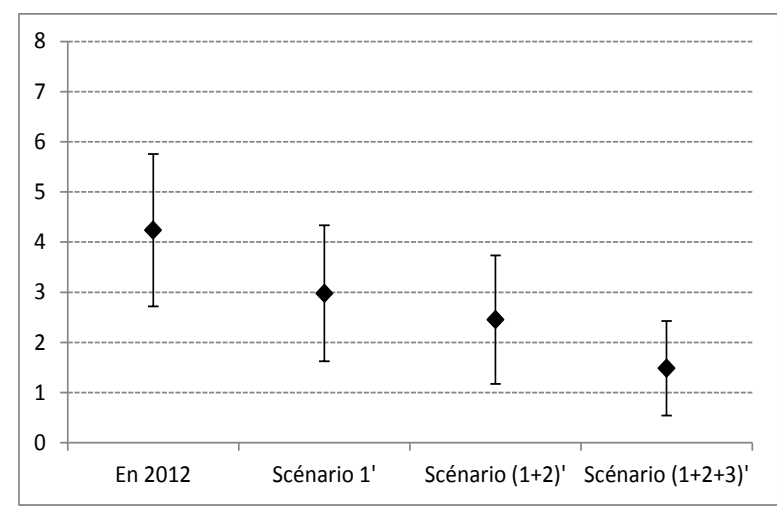

6.2 Entre 18 et 30 ans (Ref : bonne santé)

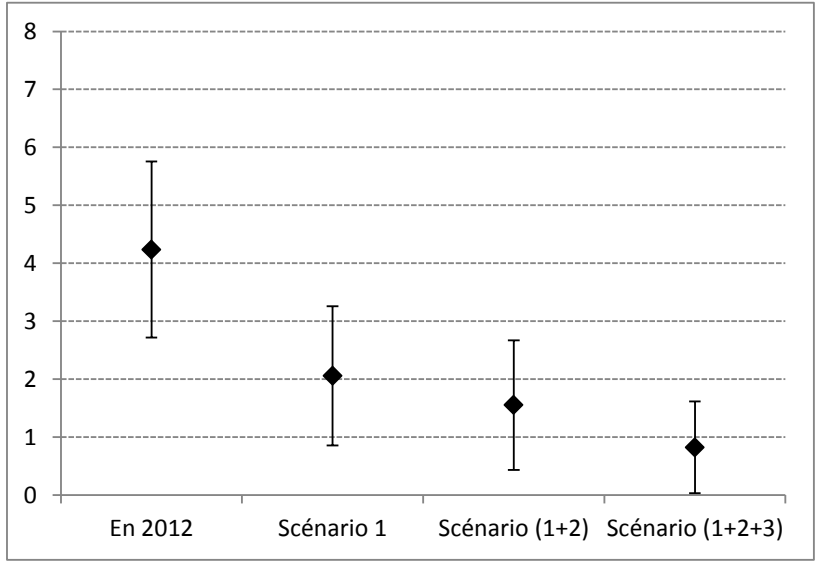

6.4 Plus de 80 ans (Ref : bonne santé)

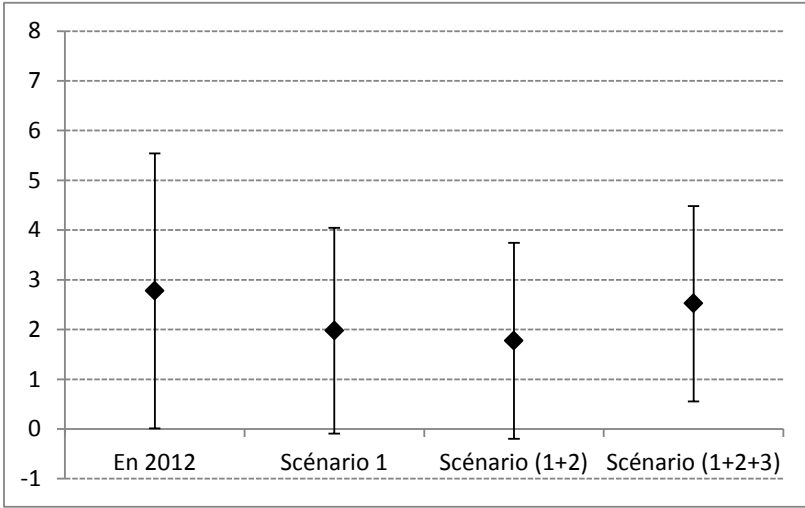

Graphiques 7 : Lien entre la non couverture et les variables socio-économiques

7.1 Retraités (Ref : en emploi)

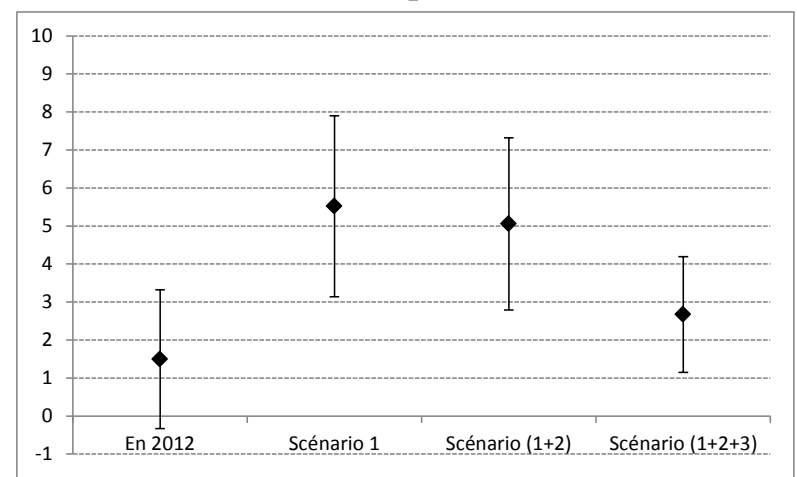

\subsection{Chômeurs (Ref : en emploi)}

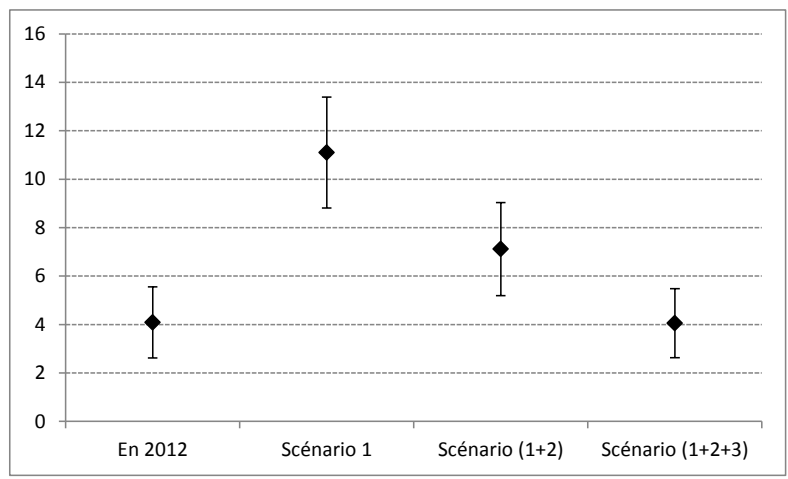


7.3 Etudiants (Ref : en emploi)

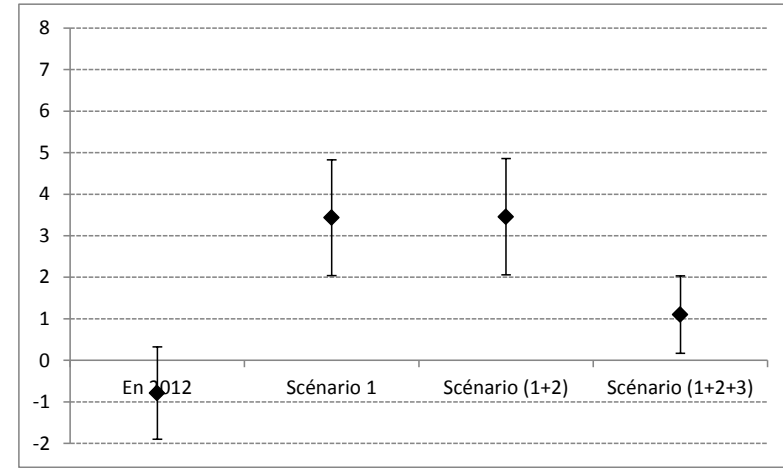

7.5 Revenu/UC inférieur à $650 €($ Ref : > 2000€)

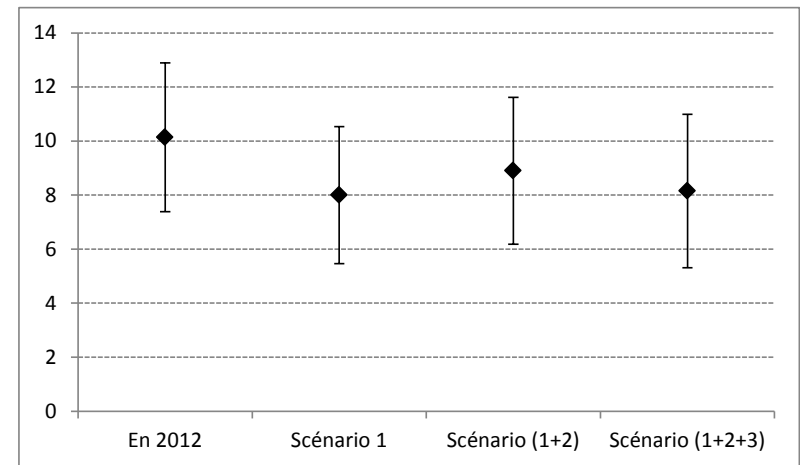

7.4 Au foyer (Ref : en emploi)

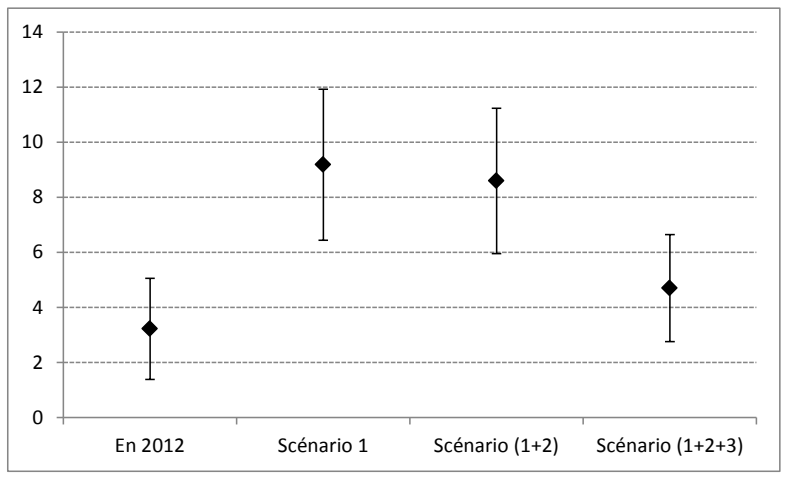

7.6 En situation de vulnérabilité sociale

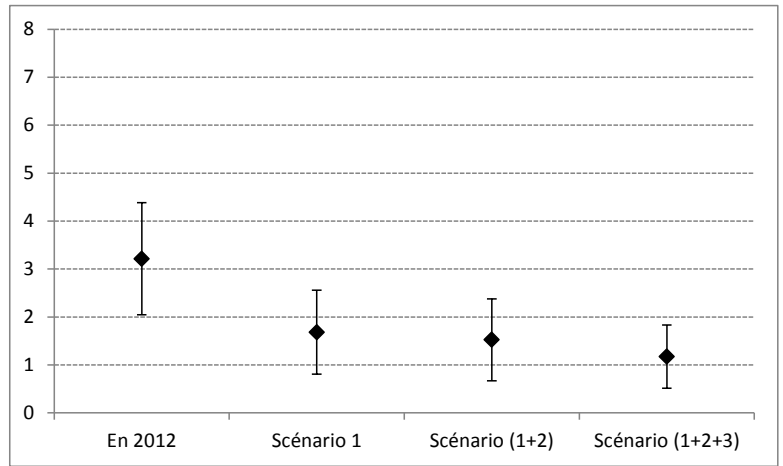

Graphiques 8 : Lien entre la non couverture et les variables de préférences

8.1. Avec une préférence pour le présent

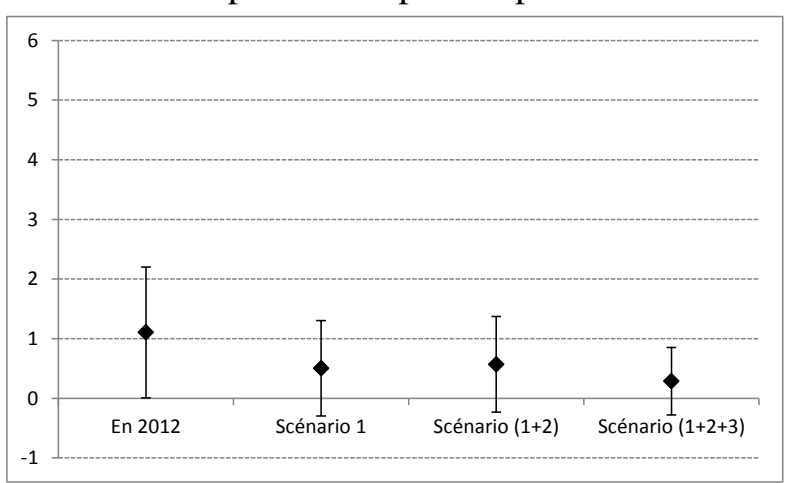

8.2. Avec une préférence à l'égard du risque

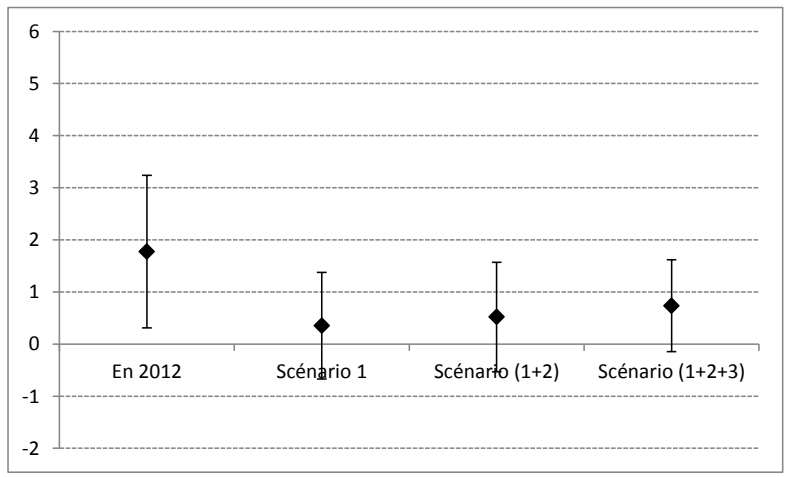




\section{Discussion}

Les analyses menées dans ce travail permettent de mettre en évidence des résultats qui questionnent les impacts à attendre de la généralisation de la complémentaire santé d'entreprise et de la portabilité tant en terme d'efficacité pour atteindre la généralisation de la complémentaire santé en France que d'équité dans l'accès à la complémentaire. En effet, le taux de non couverture, qui s'élève à $5 \%$ en 2012, est estimé à $4 \%$ après la généralisation de la complémentaire santé d'entreprise et à 3,7 \% après la portabilité. Par ailleurs, nous montrons que les plus de 70 ans, les inactifs, les individus en mauvaise santé et ceux dont le revenu par UC est le plus bas resteront, après l'ANI, les plus souvent concernés par la non couverture par une complémentaire santé. Les analyses toutes choses égales par ailleurs permettent en outre de mettre en évidence que même si l'ANI permettra de réduire l'association entre vulnérabilité sociale et non couverture, elle ne parviendra pas à éliminer les inégalités d'accès à la complémentaire santé selon le revenu. De même, les résultats mettent en évidence un renforcement du lien toutes choses égales par ailleurs entre la non couverture et le fait d'être inactifs ou au chômage alors même que les chômeurs sont, en partie, directement concernés par la portabilité de la complémentaire santé d'entreprise. Cela s'explique par la restriction de la portabilité aux seuls chômeurs de courte durée bénéficiant d'une allocation chômage. Or, des travaux récents de la DARES ont mis en évidence l'importance du nombre de chômeurs qui ne touchent pas d'indemnité (Grangier et Vinceneux, 2014). Enfin, nous montrons que les préférences du risque et du temps, qui sont significativement liées à la non couverture en 2012, suggérant que celle-ci résulte en partie d'un choix éclairé, n'expliqueront plus la non couverture après l'ANI, révélant ainsi les contraintes introduites par ce dispositif pour ceux qui chez la non couverture est choisie et non subie.

Ces résultats interrogent d'autant plus ce dispositif que les différents scénarios envisagés surestiment le nombre d'individus concernés par l'ANI. En effet, pour les salariés tout d'abord, nous ne tenons pas compte d'un éventuel délai de carence. Par ailleurs, la diminution de la non couverture chez les 18/30 ans est conditionnée au fait que les CDD de moins de 6 mois ne refusent pas d'adhérer à la complémentaire santé d'entreprise alors même que la dispense d'adhésion concerne tous les CDD de moins d'un an. Il serait intéressant par ailleurs de pouvoir étendre cette hypothèse à l'ensemble des salariés qui bénéficient d'une dispense d'adhésion, notamment pour ceux pour lesquels la cotisation peut représenter plus de $10 \%$ de leur revenu qui sont par ailleurs potentiellement les plus souvent concernés par le fait de ne pas bénéficier d'une complémentaire santé. Pour les chômeurs ensuite, nos analyses surestiment également les individus concernés par la portabilité dans la mesure où il n'est pas possible d'identifier ceux qui ne touchent pas d'allocation chômage dès lors qu'une autre personne du ménage est elle-même concernée par des allocations. De plus, nous considérons que tous les chômeurs de moins d'un an sont concernés par la portabilité alors même que la durée de ce droit est conditionnée à la durée d'ancienneté dans le dernier contrat de travail et que, dans le cas d'une demande d'avance de frais qui peut s'avérer particulièrement onéreuse, certains chômeurs peuvent refuser de conserver la 
complémentaire santé d'entreprise après leur départ de l'entreprise. Notons que seul le troisième scénario, qui tient compte simultanément de la généralisation, de la portabilité et de l'inclusion des ayants droit, permet de ne pas augmenter le lien entre la non couverture et le fait d'être au chômage ou inactif. Cependant, rappelons que l'Enquête Santé et Protection Sociale ne permet pas d'interroger les individus affiliés à une Section Locale Mutualiste (SLM) et vivant seuls, ce qui est le cas de certains étudiants et potentiellement des jeunes entrant sur le marché du travail. Enfin, ces simulations ont été obtenues sous l'hypothèse d'exogénéité de la généralisation et de la portabilité de la complémentaire santé d'entreprise. Or, on ne peut exclure que le fait que ce dispositif modifie les comportements d'offre de travail.

Au final et malgré ces hypothèses de travail relativement larges, les résultats de ces simulations montrent que le dispositif de l'ANI n'aura qu'un effet modeste sur la généralisation de la complémentaire santé en France et ne permettra pas d'atteindre l'objectif général de réduction des inégalités de santé puisque ce dispositif risque d'augmenter la concentration de la non couverture parmi les populations les plus fragiles, c'est-à-dire les plus pauvres ou les plus risquées compte tenu de leur état de santé. De plus ce dispositif risque également de réduire le bien-être des personnes pour qui la non couverture était un choix issu d'un arbitrage entre les dépenses de complémentaire et les bénéfices attendus, compte tenu de leur risque et de leurs préférences. Notons cependant que, à garanties équivalentes, la baisse du coût entre un contrat individuel et un contrat collectif peut modifier la décision de non couverture des individus à préférences données. Pour compléter ce travail, il sera important de suivre les effets de la mise en place effective de l'ANI sur la non couverture mais également d'évaluer l'influence de ce dispositif sur la modification du risque assurantiel entre le marché de la complémentaire santé individuelle et celui de la complémentaire santé collective. Enfin, l'évolution des niveaux de garanties des contrats collectifs devra également être suivie, les petites entreprises n'ayant pas les mêmes capacités de négociations et de financement que celles des grandes, qui sont, en 2009, les plus souvent concernés par les contrats de groupe (Perronnin et al., 2012).

\section{Bibliographie}

Arnould M.L et Vidal G. (2008), «Typologie des contrats les plus souscrits auprès des complémentaires santé en $2006 »$, Etudes et résultats nº63.

Arrow K.-J. (1963) : «Uncertainty and the welfare of medical care”. American Economic Review, 53 (5), pp. 941-973.

Barsky, Robert B., F. Thomas Juster, Miles S. Kimball, and Matthew D. Shapiro. 1997."Preference Parameters and Behavioral Heterogeneity: An Experimental Approach to the Health and Retirement Survey.” Quarterly Journal of Economics 112(2): 537-580.

Buchmueller TC, Couffinhal A, Grignon M, Perronnin M. (2004), Access to physician services: does supplemental insurance matter? Evidence from France. Health Economics 13(7): 669-687. 
Buchmueller TC, Couffinhal A. (2004), Private health insurance in France. OECD Health Working Paper No. 12. http://www.oecd.org/dataoecd/35/11/30455292.pdf.

Butler J. (1999): «Estimating Elasticities of Demand for Private Health Insurance in Australia», National Centre for Epidemiology and Population Health; Cenberra, ANU.

Célant N., Guillaume S., Rochereau T. (2014). «Enquête sur la santé et la protection sociale 2012 ». Les rapports de $l^{\prime} \operatorname{Irdes} \mathrm{n}^{\circ} 556$.

Doiron D., Jones G., Savage E. (2008), « Healthy, Wealthy and Insured? The Role of Self-Assessed Health in the Demand for Private Health Insurance », Health Economics, 17, 3 : 317-334

Dourgnon P., Jusot F., Fantin R. (2012), "Payer nuit gravement à la santé : une étude de l'impact du renoncement financier aux soins sur l'état de santé", Economie Publique, 28-29 : 123-147.

Franc C., Pierre A. (2013) « Generalization of Private Health Insurance offered by employers », The Health Systems and Policy monitor, avril 2013.

Garnero M., Le Palud V. (2013), «Les contrats les plus souscrits auprès des complémentaires santé en $2010 »$, Etudes et Résultats, 837.

Grangier J., Vinceneux K. (2014) : «Les demandeurs d'emploi non indemnisables par l'assurance chômage », Dares analyses n 37 .

Grignon M., Kambia-Chopin B. (2009), "Income and the demand for complementary health insurance in France", Document de travail IRDES, 24.Hopkins, S. and M. P. Kidd (1996), « The determinants of the demand for private health insurance under medicare », Applied Economics, 1623 - 1632, Volume 28, Number 12/December 1, 1996.

Jusot F. (2013), "Les inégalités de recours aux soins : bilan et évolution", Revue d'Epidémiologie et de Santé Publique, 61S : S163-S169.

Jusot F. (2014), "La complémentaire santé : une source d'inégalités face à la santé ?", In : Les inégalités de santé. Les Tribunes de la santé - Sève, 43 : 69-78.

Jusot F., Perraudin C., Wittwer J. (2012), "L'accessibilité financière à la complémentaire santé en France : les résultats de l'enquête Budget de Famille 2006", Economie et Statistique, 450 : 29-46.

Jusot F., Wittwer J. (2009), «L'accès financier aux soins en France : bilan et perspective », Regards croisés sur l'économie, 5, 1:102-109.

Kambia-Chopin B., Perronnin M., Pierre A., Rochereau T. (2008), «Les contrats complémentaires individuels : quel poids dans le budget des ménages ?» in: Enquête sur la Santé et la protection sociale 2006 (ESPS 2006), Rapport Irdes n 1701 : 43-55.

Monheit, A., Vistnes, J. (2008),. « Health Insurance Enrollment Decisions: Preferences for Coverage, Worker Sorting, and Insurance Take Up », Inquiry, 45 (2) : 153-67. 
Perronnin M., Pierre A., Rochereau T. (2011), «La complémentaire santé en France en 2008 : une large diffusion mais des inégalités d'accès », Questions d'économie de la Santé, IRDES, nº161.

Perronnin M., Pierre A., Rochereau T. (2012), «Panorama de la complémentaire santé collective en France en 2009 et opinions des salariés sur le dispositif », Questions d'économie de la Santé, IRDES, $\mathrm{n}^{\circ} 181$

Saliba B., Ventelou B. (2007) : «Complementary health insurance in France : Who pay? Why? Who will suffer from public disengagement?", Health policy 81(2007) 166-182.

Touraine M. (2014), "Health inequalities and France's national health strategy", Lancet, 383, 9923: 1101-1102.

Zaidman C., Roussel R eds (2014), Comptes nationaux de la santé 2013 - édition 2014, Drees, Collection Études et statistiques. 


\section{Annexes}

Tableau 4 : Proportion de non couverts observés en 2012 et simulés selon les scénarios $(1),(1+2)$ et $(1+2+3)$

\begin{tabular}{|c|c|c|c|c|c|}
\hline & \\
\hline & En 2012 & $\begin{array}{c}\text { Scénario } \\
\text { (1) }\end{array}$ & $\begin{array}{c}\text { Scénario } \\
(1+2)\end{array}$ & $\begin{array}{l}\text { Scénario } \\
(1+2+3)\end{array}$ & Effectifs \\
\hline \multicolumn{6}{|l|}{ Sexe } \\
\hline Hommes & 5,8 & 4,5 & 4,2 & 3,1 & 11434 \\
\hline Femmes/Hommes au foyer & 4,3 & 3,5 & 3,3 & 2,3 & 11612 \\
\hline \multicolumn{6}{|l|}{ Age } \\
\hline-18 ans & 4,1 & 4,1 & 4,1 & 1,3 & 5383 \\
\hline De 18 à 30 ans & 8,0 & 5,6 & 4,5 & 3,2 & 3760 \\
\hline De 31 à 40 ans & 4,9 & 3,2 & 2,5 & 1,9 & 2693 \\
\hline De 41 à 50 ans & 4,6 & 2,7 & 2,6 & 2,0 & 3408 \\
\hline De 51 à 60 ans & 4,2 & 3,0 & 2,9 & 2,4 & 3320 \\
\hline De 61 à 70 ans & 4,2 & 4,1 & 4,1 & 3,8 & 2458 \\
\hline De 71 à 80 & 4,3 & 4,3 & 4,3 & 4,3 & 1271 \\
\hline+ de 80 ans & 6,8 & 6,8 & 6,8 & 6,8 & 688 \\
\hline \multicolumn{6}{|l|}{ Santé perçue } \\
\hline Très bon & 4,6 & 3,5 & 3,3 & 1,8 & 5007 \\
\hline Bon & 4,0 & 3,1 & 2,8 & 2,1 & 7285 \\
\hline Assez bon & 4,8 & 3,9 & 3,7 & 3,1 & 3639 \\
\hline Mauva is & 9,9 & 8,8 & 8,8 & 8,1 & 1005 \\
\hline \multicolumn{6}{|l|}{ ALD } \\
\hline Oui & 6,3 & 5,8 & 5,6 & 5,2 & 3249 \\
\hline Non & 4,8 & 3,7 & 3,4 & 2,2 & 19647 \\
\hline \multicolumn{6}{|l|}{ Maladie Chronique } \\
\hline Oui & 5,0 & 4,4 & 4,2 & 3,8 & 5145 \\
\hline Non & 4,5 & 3,4 & 3,1 & 2,0 & 11284 \\
\hline \multicolumn{6}{|l|}{ Limitations fonctionnelles } \\
\hline Fortement limité & 8,2 & 7,7 & 7,5 & 7,1 & 1041 \\
\hline Faiblement limité & 4,9 & 4,2 & 4,1 & 3,6 & 2609 \\
\hline Pas limité & 4,3 & 3,3 & 3,0 & 2,0 & 13196 \\
\hline \multicolumn{6}{|l|}{ Statut vis-à-vis de l'emploi } \\
\hline Actifs & 3,7 & 1,2 & 1,2 & 0,9 & 9370 \\
\hline Retraités & 4,6 & 4,6 & 4,6 & 4,4 & 4063 \\
\hline Chômeurs & 13,7 & 13,7 & 9,1 & 7,2 & 1480 \\
\hline Enfants scolarisés, bébés & 4,3 & 4,3 & 4,3 & 1,6 & 6573 \\
\hline Femmes/Hommes au foyer & 8,9 & 8,9 & 8,9 & 6,7 & 960 \\
\hline Autres inactifs & 11,6 & 11,6 & 11,6 & 10,5 & 550 \\
\hline \multicolumn{6}{|l|}{ Revenu par UC } \\
\hline Revenu/UC $<=650$ & 14,1 & 13,1 & 12,7 & 10,2 & 1533 \\
\hline Revenu/UC entre $651 €$ et $1000 €$ & 8,6 & 7,4 & 6,8 & 4,7 & 3102 \\
\hline Revenu/UC entre $1001 €$ et $1400 €$ & 4,4 & 3,5 & 3,2 & 2,4 & 4168 \\
\hline Revenu/UC entre $1401 €$ et $2000 €$ & 2,7 & 1,7 & 1,7 & 1,4 & 4516 \\
\hline Revenu/UC entre $2001 €$ et $3000 €$ & 1,6 & 0,7 & 0,7 & 0,4 & 2587 \\
\hline Revenu/UC > 3000€ & 3,6 & 2,9 & 2,4 & 1,5 & 1023 \\
\hline \multicolumn{6}{|l|}{ Indicateur de vulnérabilité sociale ( 15 ans et +) } \\
\hline En situation de vulnérabilité & 9,0 & 6,9 & 6,3 & 5,6 & 2441 \\
\hline Pas en situation de vulnérabilité & 3,6 & 2,8 & 2,6 & 2,1 & 10343 \\
\hline \multicolumn{6}{|l|}{ Soutien matériel dans l'entourage (15 ans et +) } \\
\hline Avec un soutien matériel & 4,1 & 3,0 & 2,8 & 2,3 & 9171 \\
\hline Sans soutien matériel & 7,1 & 5,7 & 5,4 & 4,8 & 2151 \\
\hline Avec un soutien matériel mais sans oser demander & 5,1 & 4,2 & 3,9 & 3,4 & 1347 \\
\hline \multicolumn{6}{|l|}{ Aversion au risque (15 ans et + ) } \\
\hline Risquophobes & 4,4 & 3,5 & 3,2 & 2,7 & 11633 \\
\hline Risquophiles & 8,1 & 5,0 & 4,7 & 4,0 & 1057 \\
\hline \multicolumn{6}{|l|}{ Préférences temporelles (15 ans et + ) } \\
\hline Préférence pourle futur & 4,2 & 3,2 & 3,0 & 2,5 & 11018 \\
\hline Préférence pourle présent & 7,3 & 5,7 & 5,6 & 4,9 & 1634 \\
\hline Motifs de non couverture ( 15 ans et + non couverts) & & & & & \\
\hline Pris en charge à $100 \%$ & 100 & 93,9 & 93,9 & 88,1 & 77 \\
\hline N'a pas les moyens & 100 & 80,7 & 74,1 & 64,8 & 248 \\
\hline Ne souhaite pas être couvert & 100 & 63,9 & 62,2 & 61,1 & 52 \\
\hline En cours d'adhésion & 100 & 62,2 & 54,1 & 35,8 & 53 \\
\hline N'ypense pas & 100 & 66,4 & 66,4 & 56,1 & 41 \\
\hline Autre & 100 & 84,0 & 76,6 & 58,6 & 61 \\
\hline Total de la population & 5,0 & 4,0 & 3,7 & 2,7 & 23046 \\
\hline Total des 15 ans et + & 5,2 & 4,0 & 3,7 & 3,0 & 18561 \\
\hline
\end{tabular}

